International Journal of Theoretical and Applied Finance

Vol. 7, No. 3 (2004) 337-384

(C) World Scientific Publishing Company

\title{
THE SPECTRAL DECOMPOSITION OF THE OPTION VALUE
}

\author{
VADIM LINETSKY \\ Department of Industrial Engineering and Management Sciences \\ McCormick School of Engineering and Applied Sciences \\ Northwestern University, 2145 Sheridan Road, Evanston, IL 60208, USA \\ linetsky@iems.northwestern.edu \\ http://www.iems.northwestern.edu/ linetsky
}

Received 11 July 2003

Accepted 12 November 2003

\begin{abstract}
This paper develops a spectral expansion approach to the valuation of contingent claims when the underlying state variable follows a one-dimensional diffusion with the infinitesimal variance $a^{2}(x)$, drift $b(x)$ and instantaneous discount (killing) rate $r(x)$. The Spectral Theorem for self-adjoint operators in Hilbert space yields the spectral decomposition of the contingent claim value function. Based on the Sturm-Liouville (SL) theory, we classify Feller's natural boundaries into two further subcategories: non-oscillatory and oscillatory/non-oscillatory with cutoff $\Lambda \geq 0$ (this classification is based on the oscillation of solutions of the associated SL equation) and establish additional assumptions (satisfied in nearly all financial applications) that allow us to completely characterize the qualitative nature of the spectrum from the behavior of $a, b$ and $r$ near the boundaries, classify all diffusions satisfying these assumptions into the three spectral categories, and present simplified forms of the spectral expansion for each category. To obtain explicit expressions, we observe that the Liouville transformation reduces the SL equation to the one-dimensional Schrödinger equation with a potential function constructed from $a, b$ and $r$. If analytical solutions are available for the Schrödinger equation, inverting the Liouville transformation yields analytical solutions for the original SL equation, and the spectral representation for the diffusion process can be constructed explicitly. This produces an explicit spectral decomposition of the contingent claim value function.
\end{abstract}

Keywords: Option pricing; barrier options; diffusion processes; spectral expansion; Sturm-Liouville equation.

\section{Introduction}

Many problems in finance can be reduced to the following basic valuation problem. Assume that, under the risk-neutral probability measure, the underlying economic uncertainty follows a continuous-time Markov process $\left\{X_{t}, t \geq 0\right\}$ taking values in some state space $E$. Consider a cash flow or payoff $f$ at some time $t \geq 0$ and contingent on the state of the process at $t, f=f\left(X_{t}\right)$, where $f=f(x)$ is a realvalued function on $E$. The time-zero present value or price of this payoff can then be represented as the risk-neutral expectation of the discounted payoff:

$$
V(t, x)=\mathbb{E}_{x}\left[e^{-\int_{0}^{t} r\left(X_{u}\right) d u} f\left(X_{t}\right)\right]=:\left(\mathcal{P}_{t} f\right)(x),
$$


where $r=r(x)$ is a non-negative function on $E$ (instantaneous discount rate). The subscript $x$ in the expectation operator $\mathbb{E}_{x}$ indicates that $X_{0}=x \in E$. In the standard Markovian set-up, one considers a Banach space $B(E)$ of real-valued, bounded Borel-measurable payoff functions on $E$. Then the valuation relationship defines a strongly continuous semigroup of contractions $\left\{\mathcal{P}_{t}, t \geq 0\right\}$ in $B(E)$ (pricing semigroup).

One generally has four possible approaches to compute the price or value function $V=V(t, x)$. Firstly, one may try to compute the expectation analytically. Secondly, one can simulate the underlying Markov process and estimate the expectation on a finite set of simulated sample paths (e.g., [6]). Thirdly, one can construct a Markov chain approximation for the continuous-time process $X$. Binomial and trinomial lattices popular in finance belong to this category. Finally, let $\mathcal{G}$ be the infinitesimal generator of the pricing semigroup. Then the value function $V(t, x)=\left(\mathcal{P}_{t} f\right)(x)$ solves the Cauchy problem for the evolution equation:

$$
V_{t}=\mathcal{G} V \text { for } t>0, \text { with } V(0, x)=f(x) .
$$

Approximate solutions to this evolution equation can be constructed by numerical methods. Generally, Monte Carlo simulation, Markov chain approximations and numerical evolution equation methods are the main numerical approaches to compute the value function in this general Markov process/Banach space semigroup set-up.

A significant analytical simplification is possible if the Hilbert space structure is available and the pricing semigroup is self-adjoint. In particular, suppose there is a (finite or infinite) measure $\mathfrak{m}$ on $E$ such that the pricing semigroup $\left\{\mathcal{P}_{t}, t \geq 0\right\}$ restricted to $B(E) \cap L^{2}(E, \mathfrak{m})$ extends uniquely to a strongly continuous semigroup of self-adjoint contractions in the real Hilbert space $L^{2}(E, \mathfrak{m})$ with inner product $(f, g)=\int_{E} f g d \mathfrak{m}$, i.e.,

$$
\left(\mathcal{P}_{t} f, g\right)=\left(f, \mathcal{P}_{t} g\right) \text { for any } t \geq 0 \text { and } f, g \in L^{2}(E, \mathfrak{m}) .
$$

Then the infinitesimal generator $\mathcal{G}$ is a (generally unbounded) self-adjoint operator in $L^{2}(E, \mathfrak{m})$. In this special case one can invoke the Spectral Theorem for self-adjoint operators in Hilbert space (e.g., $[22,81])$ to obtain a unique spectral representation for the pricing semigroup. In a number of applications one is able to compute the spectral representation explicitly, thus generating a closed-form solution for the value function.

The self-adjointness requirement restricts the class of Markov processes to symmetric Markov processes (e.g., [20, Section XIII.4] and [27, Section 1.3]). When the process is a one-dimensional diffusion on some interval $I \subseteq \mathbb{R}$ (open or closed, finite or infinite) with the (finite or infinite) speed measure $\mathfrak{m}$ and appropriate boundary conditions at the end-points of the interval, the pricing semigroup is selfadjoint in $L^{2}(I, \mathfrak{m})$ without any further conditions and the Spectral Theorem yields a spectral decomposition of the value function. For multi-dimensional diffusions, the self-adjointness restricts the form of the drift vector (see, e.g., [27]). For jump 
processes, the self-adjointness restricts the class of processes to jump processes with symmetric jump size distributions.

In this paper we focus on one-dimensional diffusions. Applications of spectral theory to one-dimensional diffusions go back to the fundamental paper by McKean [69] (see also Elliot [23], Ito and McKean [42, Section 4.11] and, for more recent references, Kent [48, 49] and Langer and Schenk [51]). Based on the work of Feller, McKean constructed a spectral representation for a general one-dimensional diffusion. At around the same time Karlin and McGregor [45] constructed spectral representations for birth-and-death processes. When there are no natural boundaries, Elliot [23] and McKean [69] proved that the spectrum of the infinitesimal generator is purely discrete (see also Kent [48]). However, when one or both boundaries are natural the situation is more complicated. The infinitesimal generator may (but not always does) have some non-empty continuous spectrum. Moreover, it may be non-simple.

The goal of the present paper is to develop applications of the spectral theory of one-dimensional diffusions to finance. In Sec. 2 we state the valuation problem when the underlying variable follows a one-dimensional diffusion. We interpret the nonnegative discount rate as a killing rate for the underlying diffusion and the pricing semigroup as a transition semigroup for the diffusion with killing. In Sec. 3 we recall McKean's general form of the spectral representation for one-dimensional diffusions specialized to our setting. If natural boundaries are allowed, it is expressed in terms of a spectral matrix, a Borel measure from $(-\infty, 0]$ to $2 \times 2$ symmetric non-negative definite matrices. Without additional assumptions, it is not in general possible to explicitly characterize discrete and continuous parts of the spectrum. This is a drawback for financial applications. In applications, one would like to be able to explicitly characterize the spectrum.

In Sec. 4 we take up the issue of determining a set of simplifying assumptions that allow one to completely characterize the spectrum of a diffusion with natural boundaries. Many financial diffusion models have natural boundaries, so this is an important issue for applications. First, based on the Sturm-Liouville theory applied to the negative of the infinitesimal generator, $\mathcal{A}:=-\mathcal{G}$, we classify natural boundaries into two further subcategories: non-oscillatory (NONOSC) and oscillatory/non-oscillatory (O-NO) with cutoff $\Lambda \geq 0$. This classification is based on the oscillation of solutions of the Sturm-Liouville equation $\mathcal{A} u=\lambda u$. NONOSC natural boundaries do not generate any continuous spectrum, while O-NO natural boundaries generate some continuous spectrum above the cutoff value $\Lambda$. If there is only one O-NO natural boundary, then the continuous spectrum is simple. If both boundaries are O-NO natural, then the continuous spectrum is not simple. Without any further assumptions, the continuous spectrum may have a complicated structure. In particular, it may consist of an infinite sequence of disjoint intervals separated by gaps. Furthermore, eigenvalues may be present in the gaps or embedded in the continuous spectrum. However, adding several additional assumptions (Assumptions 2-4) that are satisfied in nearly all financial applications yields a 
drastic simplification of the structure of the spectrum and allows us to classify all diffusions satisfying these assumptions into three spectral categories and present in Sec. 5 simplified forms of the spectral representation specific to each category. In the Spectral Category I (no O-NO natural boundaries), the spectrum is purely discrete. In the Spectral Category II (one O-NO natural boundary with cutoff $\Lambda$ ), there is a simple purely absolutely continuous spectrum above the cutoff value and purely discrete spectrum below the cutoff value (the latter may be empty). In the Spectral Category III (two O-NO natural boundaries with cutoffs $\Lambda_{1}$ and $\Lambda_{2}$ ), there is a multiplicity-two purely absolutely continuous spectrum above $\max \left\{\Lambda_{1}, \Lambda_{2}\right\}$, simple purely absolutely continuous spectrum between $\min \left\{\Lambda_{1}, \Lambda_{2}\right\}$ and $\max \left\{\Lambda_{1}, \Lambda_{2}\right\}$, and purely discrete spectrum below $\min \left\{\Lambda_{1}, \Lambda_{2}\right\}$ (the latter may be empty). The results of Secs. 4 and 5 allow one to read off the qualitative nature of the spectrum of $\mathcal{A}$ in $L^{2}(E, \mathfrak{m})$ from the functional forms of the diffusion coefficient $a(x)$, drift $b(x)$ and killing (discount) rate $r(x)$ and give the recipe for constructing the spectral representation explicitly.

In order to determine the spectral representation explicitly, one needs explicit expressions for solutions of the Sturm-Liouville equation. The Liouville transformation reduces the Sturm-Liouville equation associated with a one-dimensional diffusion with diffusion coefficient $a(x)$, drift $b(x)$ and killing rate $r(x)$ to the onedimensional Schrödinger equation with a potential function $Q(x)$ constructed from $a(x), b(x)$ and $r(x)$. Correspondingly, we say that the Sturm-Liouville equation is reducible to the Schrödinger equation with potential $Q$. If analytical solutions are available for the Schrödinger equation, inverting the Liouville transformation yields analytical solutions for the original Sturm-Liouville equation, and the spectral representation for the diffusion process can be constructed explicitly.

In the second part of the paper, Sec. 6 , we survey some analytically tractable Schrödinger equations, associated diffusion processes and their applications in finance. While, due to the space limitations, this survey is not exhaustive, it does include many of the most important analytically tractable financial models. Table 2 in the Conclusion summarizes the analytically tractable models surveyed in Sec. 6 . The Appendix states the Spectral Theorem for self-adjoint semigroups in Hilbert space and summarizes some definitions and results from spectral theory needed in the paper.

To conclude this introduction, we give an (incomplete) reference list of recent works which have employed spectral expansions in finance. Lewis [56] and Goldstein and Keirstead [33] apply the eigenfunction expansion approach to the pricing of bonds when the short-rate process follows a one-dimensional diffusion. Lewis [57] applies the eigenfunction expansion approach to the pricing of options on stocks that pay continuous dividends at a constant dollar rate and the pricing of bonds under Merton's short-rate process with non-linear drift. Lewis [58] applies the eigenfunction expansion approach to the analysis of stochastic volatility models. Lipton $[66,67]$ and Lipton and McGhee [68] extensively apply spectral expansions to the pricing of vanilla and barrier options under various alternative specifications of the 
local volatility function (including the quadratic model) as well as under stochastic volatility (see also [3] for various alternative specifications of the local volatility function). He et al. [38] apply spectral expansions to the pricing of double lookback options. Davydov and Linetsky [19] develop spectral expansions for singleand double-barrier options under the CEV process, as well as interest rate barrier options in the CIR model. Linetsky [60,61] develops spectral expansions for arithmetic Asian options in the Black-Scholes model. Gorovoi and Linetsky [34] solve Black's model of interest rates as options via spectral expansions. Linetsky [62] develops spectral expansions for lookback options. Hansen, Scheinkman, and Touzi [37], Florens, Renault, and Touzi [26] and Larsen and Sorensen [52] apply spectral expansions to the estimation of diffusion models from time-series data.

\section{The Valuation Problem When the Underlying Financial Variable Follows a One-Dimensional Diffusion}

Assume that, under the risk-neutral probability measure, the underlying economic uncertainty follows a one-dimensional, time-homogeneous regular diffusion $\left\{X_{t}, t \geq\right.$ $0\}$ whose state space $E$ is some interval $I \subseteq \mathbb{R}$ with endpoints $e_{1}$ and $e_{2},-\infty \leq$ $e_{1}<e_{2} \leq \infty$, and the infinitesimal generator

$$
(\mathcal{G} f)(x)=\frac{1}{2} a^{2}(x) f^{\prime \prime}(x)+b(x) f^{\prime}(x), \quad x \in\left(e_{1}, e_{2}\right),
$$

acting on functions on $I$ subject to appropriate regularity and boundary conditions. We assume that the diffusion coefficient $a(x)$ is continuous and strictly positive on the open interval $\left(e_{1}, e_{2}\right)$ and drift $b(x)$ is continuous on $\left(e_{1}, e_{2}\right)$. The infinitesimal generator can be re-written as

$$
(\mathcal{G} f)(x)=\frac{1}{\mathfrak{m}(x)}\left(\frac{f^{\prime}(x)}{\mathfrak{s}(x)}\right)^{\prime}, \quad x \in\left(e_{1}, e_{2}\right),
$$

where $\mathfrak{s}(x)$ and $\mathfrak{m}(x)$ are the scale and speed densities (see [5, Chapter II]) for details on one-dimensional diffusions):

$$
\mathfrak{s}(x):=\exp \left(-\int^{x} \frac{2 b(y)}{a^{2}(y)} d y\right), \quad \mathfrak{m}(x):=\frac{2}{a^{2}(x) \mathfrak{s}(x)} .
$$

Both $\mathfrak{s}(x)$ and $\mathfrak{m}(x)$ are continuous and strictly positive on $\left(e_{1}, e_{2}\right)$. The endpoints $e_{i}, i=1,2$, are either natural, entrance, exit, or regular boundaries for the diffusion $X$. In this paper we consider two types of regular boundaries: killing or instantaneously reflecting. For regular instantaneously reflecting boundaries, the boundary point $e$ is included in the state space $I$ and $(\mathcal{G} f)(e):=\lim _{x \rightarrow e}(\mathcal{G} f)(x)$. For natural, entrance, exit and regular killing boundaries, the boundary point is not included in $I$. For exit and regular killing boundaries, the process $X$ is sent to a cemetery state $\partial$ at the first hitting time of the boundary.

Consider a cash flow or payoff $f$ at some time $t \geq 0$ and contingent on the state of the process at $t, f=f\left(X_{t}\right)$, where $f=f(x)$ is a real-valued function on 
$I$ subject to appropriate regularity conditions. By convention, a function defined on $I$ takes the value 0 at the cemetery state, $f(\partial)=0$ (for credit risk applications one may assign a certain non-zero "recovery" payoff to the cemetery state). The time-zero present value or price can be represented as a risk-neutral expectation of the discounted payoff:

$$
V(t, x)=\mathbb{E}_{x}\left[e^{-\int_{0}^{t} r\left(X_{u}\right) d u} f\left(X_{t}\right)\right], \quad x \in I,
$$

where the instantaneous discount rate $r=r(x)$ is non-negative and continuous on $\left(e_{1}, e_{2}\right)$.

Let $\tau$ be an exponential random variable with parameter 1 , independent of $X$, and introduce a new process $\hat{X}$ :

$$
\hat{X}_{t}:=\left\{\begin{array}{cc}
X_{t}, & \int_{0}^{t} r\left(X_{u}\right) d u<\tau \\
\partial, & \int_{0}^{t} r\left(X_{u}\right) d u \geq \tau
\end{array} .\right.
$$

The expectation (4) can be re-written in terms of $\hat{X}$ :

$$
V(t, x)=\mathbb{E}_{x}\left[f\left(\hat{X}_{t}\right) \mathbf{1}_{\{t<\hat{\zeta}\}}\right]=:\left(\mathcal{P}_{t} f\right)(x),
$$

where $\hat{\zeta}:=\inf \left\{t \geq 0: \hat{X}_{t}=\partial\right\}$ is the lifetime of $\hat{X}$ and, by convention, $f(\partial)=0$. The process $\hat{X}$ is a diffusion with killing whose state space is $I \cup\{\partial\}$ and with the infinitesimal generator

$$
\begin{aligned}
(\hat{\mathcal{G}} f)(x) & =\frac{1}{2} a^{2}(x) f^{\prime \prime}(x)+b(x) f^{\prime}(x)-r(x) f(x) \\
& =\frac{1}{\mathfrak{m}(x)}\left(\frac{f^{\prime}(x)}{\mathfrak{s}(x)}\right)^{\prime}-r(x) f(x)
\end{aligned}
$$

for $x \in\left(e_{1}, e_{2}\right)$. The discount rate $r(x)$ enters as a killing rate for $\hat{X}$. The state-price density $p(t ; x, y)$ of the asset pricing model with the underlying diffusion $X$ and the discount rate $r=r(X)$ is thus interpreted as a transition density of the diffusion with killing $\hat{X}$.

Feller's boundary classification for diffusions with killing is made as follows (e.g., $\left[5\right.$, pp. 14-15]). For any $x, y \in\left(e_{1}, e_{2}\right)$ define

$$
\mathcal{S}[x, y]:=\int_{x}^{y} \mathfrak{s}(z) d z, \mathcal{S}\left(e_{1}, y\right]:=\lim _{x \downarrow e_{1}} \mathcal{S}[x, y], \quad \mathcal{S}\left[x, e_{2}\right):=\lim _{y \uparrow e_{2}} \mathcal{S}[x, y]
$$

(the limits may be infinite). Furthermore, pick any $\epsilon \in\left(e_{1}, e_{2}\right)$ and define (we did some obvious interchanges of integrations in formulas on p. 14 of Borodin and Salminen [5] to present these formulas in this convenient for us form)

$$
\begin{array}{rlrl}
I_{1} & :=\int_{e_{1}}^{\epsilon} \mathcal{S}\left(e_{1}, x\right](1+r(x)) \mathfrak{m}(x) d x, & I_{2}:=\int_{\epsilon}^{e_{2}} \mathcal{S}\left[x, e_{2}\right)(1+r(x)) \mathfrak{m}(x) d x, \\
J_{1}:=\int_{e_{1}}^{\epsilon} \mathcal{S}[x, \epsilon](1+r(x)) \mathfrak{m}(x) d x, & J_{2}:=\int_{\epsilon}^{e_{2}} \mathcal{S}[\epsilon, x](1+r(x)) \mathfrak{m}(x) d x .
\end{array}
$$


The boundary $e_{i} \in\left\{e_{1}, e_{2}\right\}$ is said to be regular if $I_{i}<\infty$ and $J_{i}<\infty$, exit if $I_{i}<\infty$ and $J_{i}=\infty$, entrance if $I_{i}=\infty$ and $J_{i}<\infty$, or natural if $I_{i}=\infty$ and $J_{i}=\infty$. Note that adding the killing rate $r(x) \geq 0$ may change the nature of the boundaries, i.e., the processes $X$ and $\hat{X}$ will, in general, have different boundary classifications. A natural boundary for $X$ will remain natural for $\hat{X}$. An exit boundary for $X$ will either remain exit or become natural for $\hat{X}$. An entrance boundary for $X$ will either remain entrance or become natural for $\hat{X}$. A regular boundary for $X$ will either remain regular or become exit or natural for $\hat{X}$ (it cannot become entrance). To give an example, consider Brownian motion on the positive half-line with the regular boundary at the origin specified as a killing boundary. Killing the process at the rate $1 / x^{2}$ makes the boundary natural. Intuitively, the process will be killed before it reaches the boundary (the killing rate increases as $1 / x^{2}$ as the process approaches the origin), and the origin becomes unattainable.

In the standard Markovian set-up, one considers a Banach space $C_{b}(I)$ of realvalued, bounded continuous functions on $I$. Then the pricing operators $\mathcal{P}_{t}$ defined in Eq. (6) form a Feller semigroup (pricing semigroup) $\left\{\mathcal{P}_{t}, t \geq 0\right\}$ in $C_{b}(I)$. The domain $D(\hat{\mathcal{G}})$ of the infinitesimal generator $\hat{\mathcal{G}}$ of $\left\{\mathcal{P}_{t}, t \geq 0\right\}$ in $C_{b}(I)$ is:

$$
D(\hat{\mathcal{G}}):=\left\{f \in C_{b}(I): \hat{\mathcal{G}} f \in C_{b}(I), \text { boundary conditions at } e_{1} \text { and } e_{2}\right\} .
$$

The boundary conditions are as follows [69, p. 522], [5, pp. 16-17]). If $e \in\left\{e_{1}, e_{2}\right\}$ is exit or regular killing for $\hat{X}$, then the appropriate boundary condition at $e$ is:

$$
\lim _{x \rightarrow e} f(x)=0 \text {. }
$$

If $e \in\left\{e_{1}, e_{2}\right\}$ is entrance or regular instantaneously reflecting for $\hat{X}$, then the appropriate boundary condition at $e$ is:

$$
\lim _{x \rightarrow e} \frac{f^{\prime}(x)}{\mathfrak{s}(x)}=0 .
$$

No boundary conditions are needed at natural boundaries in addition to the requirement that $f, \hat{\mathcal{G}} f \in C_{b}(I)$.

For future convenience we summarize our assumptions about the infinitesimal parameters.

Assumption 1. The functions $a(x), b(x)$ and $r(x)$ are continuous on $\left(e_{1}, e_{2}\right)$ and $a(x)>0$ and $r(x) \geq 0$ on $\left(e_{1}, e_{2}\right)$.

\section{The General Form of the Spectral Representation for One-Dimensional Diffusions}

Our goal is to give an explicit spectral representation for the value function (6). Let $L^{2}(I, \mathfrak{m})$ be the Hilbert space of real-valued functions on $I$ square-integrable with the speed density and with the inner product

$$
(f, g)=\int_{I} f(x) g(x) \mathfrak{m}(x) d x .
$$


The Feller semigroup $\left\{\mathcal{P}_{t}, t \geq 0\right\}$ restricted to $C_{b}(I) \cap L^{2}(I, \mathfrak{m})$ extends uniquely to a strongly continuous semigroup of self-adjoint contractions in $L^{2}(I, \mathfrak{m})$ with the infinitesimal generator $\hat{\mathcal{G}}$, an unbounded self-adjoint, non-positive operator in $L^{2}(I, \mathfrak{m})[69]$ (see also [51]). We retain the same notation for the semigroup and its infinitesimal generator in $C_{b}(I)$ and $L^{2}(I, \mathfrak{m})$; this should not create any confusion since from now on we always work in $L^{2}(I, \mathfrak{m})$. The domain of $\hat{\mathcal{G}}$ in $L^{2}(I, \mathfrak{m})$ is $([69$, p. 526] and [51, p. 15]):

$$
\begin{aligned}
D(\hat{\mathcal{G}}):= & \left\{f \in L^{2}(I, \mathfrak{m}): f, f^{\prime} \in A C_{\mathrm{loc}}(I), \hat{\mathcal{G}} f \in L^{2}(I, \mathfrak{m}),\right. \\
& \text { boundary conditions at } \left.e_{1} \text { and } e_{2}\right\}
\end{aligned}
$$

where $A C_{\text {loc }}(I)$ is the space of functions absolutely continuous over each compact subinterval of $I$. If $e \in\left\{e_{1}, e_{2}\right\}$ is either exit, entrance, regular killing or regular instantaneously reflecting boundary for $\hat{X}$, then the boundary condition at $e$ is as described in Sec. 2. If $e \in\left\{e_{1}, e_{2}\right\}$ is natural and the speed density is integrable near $e,\left|\int_{e}^{\epsilon} \mathfrak{m}(x) d x\right|<\infty$ for $\epsilon \in\left(e_{1}, e_{2}\right)$, then the boundary condition at $e$ is given by Eq. (9). If $\left|\int_{e}^{\epsilon} \mathfrak{m}(x) d x\right|=\infty$, then the square integrability of $f$ near $e$, $\left|\int_{e}^{\epsilon} f^{2}(x) \mathfrak{m}(x) d x\right|<\infty$ for $\epsilon \in\left(e_{1}, e_{2}\right)$, implies Eq. (8). The self-adjointness of $\hat{\mathcal{G}}$ is proved in McKean [69] (see also [51, p. 15, Theorem 3.2]).

The Spectral Theorem for self-adjoint semigroups in Hilbert space can now be applied to produce a spectral representation for $\mathcal{P}_{t} f$ for $f \in L^{2}(I, \mathfrak{m})$ and $t \geq 0$ (see Appendix A for a quick review of the spectral theory). McKean [69] (see also [42, Section 4.11]) gives a general spectral representation for the semigroup of a one-dimensional diffusion with killing in $L^{2}(I, \mathfrak{m})$. Specializing McKean's general results to our case in which the scale function, the speed measure and the killing measure are absolutely continuous with respect to the Lebesgue measure and have smooth derivatives, we have:

$$
V(t, x)=\left(\mathcal{P}_{t} f\right)(x)=\int_{I} f(y) p(t ; x, y) d y,
$$

where the transition density has the spectral representation:

$$
p(t ; x, y)=\mathfrak{m}(y) \int_{(-\infty, 0]} e^{\lambda t} \sum_{i, j=1}^{2} u_{i}(x, \lambda) u_{j}(y, \lambda) \rho_{i j}(d \lambda), \quad t>0, \quad x, y \in I,
$$

where $u_{i}(x, \lambda), i=1,2$, are solutions of the initial value problems $\left(x_{0} \in\left(e_{1}, e_{2}\right)\right.$ can be selected arbitrarily)

$$
\begin{gathered}
\left(\hat{\mathcal{G}} u_{i}\right)(x, \lambda)=\lambda u_{i}(x, \lambda), \quad x \in\left(e_{1}, e_{2}\right), \lambda \leq 0, \\
u_{1}\left(x_{0}, \lambda\right)=1, \quad \frac{u_{1}^{\prime}\left(x_{0}, \lambda\right)}{\mathfrak{s}\left(x_{0}\right)}=0, \\
u_{2}\left(x_{0}, \lambda\right)=0, \quad \frac{u_{2}^{\prime}\left(x_{0}, \lambda\right)}{\mathfrak{s}\left(x_{0}\right)}=1,
\end{gathered}
$$


and $\rho(d \lambda)=\left(\rho_{i j}(d \lambda)\right)_{i, j=1}^{2}$ is a Borel measure from $(-\infty, 0]$ to $2 \times 2$ symmetric non-negative definite matrices (spectral matrix):

$$
\rho_{11}(d \lambda) \geq 0, \quad \rho_{22}(d \lambda) \geq 0, \quad \rho_{12}(d \lambda)=\rho_{21}(d \lambda), \quad\left(\rho_{12}(d \lambda)\right)^{2} \leq \rho_{11}(d \lambda) \rho_{22}(d \lambda) .
$$

The integral in Eq. (12) converges uniformly on compact squares in $I \times I$. McKean [69] proves a number of smoothness properties for the transition density $p(t ; x, y)$.

For $f \in L^{2}(I, \mathfrak{m})$, the spectral expansion for the value function can be written in the form:

$$
V(t, x)=\left(\mathcal{P}_{t} f\right)(x)=\int_{(-\infty, 0]} e^{\lambda t} \sum_{i, j=1}^{2} u_{i}(x, \lambda) F_{j}(\lambda) \rho_{i j}(d \lambda), \quad x \in I, \quad t \geq 0,
$$

where the expansion coefficients are

$$
F_{i}(\lambda)=\int_{I} f(y) u_{i}(y, \lambda) \mathfrak{m}(y) d y
$$

and the Parseval equality holds

$$
\|f\|^{2}=\int_{(-\infty, 0]} \sum_{i, j=1}^{2} F_{i}(\lambda) F_{j}(\lambda) \rho_{i j}(d \lambda) .
$$

When there are no natural boundaries, the spectrum is simple and purely discrete ([23] and [69, Theorem 3.1]). Let $\left\{\lambda_{n}\right\}_{n=1}^{\infty}, 0 \geq \lambda_{1}>\lambda_{2}>\cdots, \lim _{n \uparrow \infty} \lambda_{n}=$ $-\infty$, be the eigenvalues of $\hat{\mathcal{G}}$ and let $\left\{\varphi_{n}\right\}_{n=1}^{\infty}$ be the corresponding eigenfunctions normalized so that $\left\|\varphi_{n}\right\|^{2}=1$. Then the spectral representation (12) for the density and the spectral expansion (17) for the value function simplify to (for $t>0$ the series (20) converges uniformly on compact squares in $I \times I)$ :

$$
\begin{gathered}
p(t ; x, y)=\mathfrak{m}(y) \sum_{n=1}^{\infty} e^{\lambda_{n} t} \varphi_{n}(x) \varphi_{n}(y), \quad x, y \in I, \quad t>0, \\
V(t, x)=\left(\mathcal{P}_{t} f\right)(x)=\sum_{n=1}^{\infty} c_{n} e^{\lambda_{n} t} \varphi_{n}(x), \quad f \in L^{2}(I, \mathfrak{m}), \quad x \in I, \quad t \geq 0, \\
c_{n}=\left(f, \varphi_{n}\right), \quad\|f\|^{2}=\sum_{n=1}^{\infty} c_{n}^{2} .
\end{gathered}
$$

When boundaries are natural, the spectrum of $\hat{\mathcal{G}}$ may have some non-empty and possibly non-simple continuous spectrum and the spectral representation has the general form $(12),(17)$. However, under some additional regularity assumptions to be described in the next Section the general form of the spectral representation can be significantly simplified.

Remark on non- $L^{2}$ payoffs. We stress that the spectral expansion (17)-(19) (or $(21)-(22)$ in case of discrete spectrum) is valid for payoffs in $L^{2}(I, \mathfrak{m})$ (note that the payoff does not have to be bounded, but it has to be square-integrable with the speed density). If the payoff is not in $L^{2}(I, \mathfrak{m})$ but the integral in Eq. (11) exists, 
one can write the value function in the form (11) with the transition probability density given by Eq. (12) (or (20) in case of discrete spectrum). However, one cannot interchange the integration over the state space $I$ and the integration over the spectrum to obtain the spectral expansion (17) (or (21)) for the value function. In fact, this situation is relatively frequent in finance applications. Among applications discussed in Sec. 6, examples of non- $L^{2}$ payoffs include call and put options in Merton's cash dividend model (Sec. 6.5.3), Asian-style call options (Sec. 6.5.2), vanilla call options and down-and-out call options on the CEV process (Sec. 6.3.4), as well as call options in the standard Black-Scholes model. When $X$ is an asset price process, in many of these applications constants and/or linear functions are not in $L^{2}(I, \mathfrak{m})$. In some cases (e.g., Asian call, CEV call, Black-Scholes call), one is able to decompose the payoff into a linear combination of an $L^{2}(I, \mathfrak{m})$-payoff (e.g., put) plus an affine position $a+b X$ (e.g., forward). The value function for the affine position is found directly, and the value function for the $L^{2}(I, \mathfrak{m})$-payoff is given by the eigenfunction expansion (a typical application of the call-put parity relationship). However, in some applications either the payoff cannot be represented as a linear combination of an affine position plus an $L^{2}(I, \mathfrak{m})$-payoff, or the value function for the affine payoff cannot be easily found directly. An example of the first type arises in Merton's cash dividend model [57]. Both call and put payoffs $(x-K)^{+}$and $(K-x)^{+}$are not square-integrable with the speed density $m(x) \propto x^{r-2} \exp (D / x)$ (for simplicity we set $\sigma=\sqrt{2}$ ). An example of the second type arises in pricing down-and-out call options under the CEV process [19]. We can decompose the down-and-out call payoff into a linear combination of the down-and-out put payoff (which is $L^{2}$ ) and a down-and-out forward position (non- $L^{2}$ ). However, we now need to determine the value function for the down-and-out forward, which is not straightforward. In both of these applications, one can start with the representation (11) for the value function, but instead of the spectral representation (12) for the transition density one uses an alternative representation as an inverse Laplace transform of a Green's function. The next steps are interchanging the integration over the state space with the Laplace inversion, integrating the Green's function against the payoff, and finally inverting the Laplace transform. The Laplace inversion leads to a spectral-type representation. However, it includes additional terms in addition to the ones in Eq. (17) that come from additional singularities in the Laplace transform in the problem with non- $L^{2}$ payoff. If one did not check the integrability condition and attempted to interchange the integrations in (11)-(12), one would lose these additional terms. See Lewis [57] for more details.

\section{The Oscillatory/Non-Oscillatory Classification of Boundaries and the Spectral Classification of One-Dimensional Diffusions}

To proceed further, we need some facts from the Sturm-Liouville theory (e.g., $[22,28,31,55,79,84,87])$. Consider a Sturm-Liouville (SL) 2nd order ordinary 
differential equation with $\lambda \in \mathbb{C}$ and $x \in\left(e_{1}, e_{2}\right)$ :

$$
(\mathcal{A} u)(x) \equiv-\frac{1}{\mathfrak{m}(x)}\left(\frac{u^{\prime}(x)}{\mathfrak{s}(x)}\right)^{\prime}+r(x) u(x)=\lambda u(x)
$$

The Sturm-Liouville operator $\mathcal{A}$ is the negative of the diffusion infinitesimal generator:

$$
\mathcal{A}:=-\hat{\mathcal{G}} .
$$

While the infinitesimal generator $\hat{\mathcal{G}}$ is non-positive, the SL operator $\mathcal{A}$ is nonnegative. Consistent with the Sturm-Liouville literature, in Secs. 4-6 we take $\lambda \geq 0$ to be the spectral parameter of the non-negative SL operator $\mathcal{A}$. This is in contrast with Sec. 3 and Appendix A where $\lambda \leq 0$ is the spectral parameter of the nonpositive infinitesimal generator $\hat{\mathcal{G}}$. Thus, the $\lambda$ in Secs. $4-6$ is the negative of the $\lambda$ of Sec. 3 and Appendix A.

The oscillatory/non-oscillatory classification based on Sturm's theory of oscillations of solutions is of fundamental importance in determining the qualitative nature of the spectrum of the SL operator. For a given real $\lambda$, Eq. (23) is oscillatory at an end-point $e$ if and only if every solution has infinitely many zeros clustering at $e$. Otherwise it is called non-oscillatory at $e$. This classification is mutually exclusive for a fixed $\lambda$, but can vary with $\lambda$. For Eq. (23), there are two distinct possibilities at each endpoint.

Theorem 1 (Oscillatory/Non-oscillatory Classification of Boundaries). Let $e \in\left\{e_{1}, e_{2}\right\}$ be an endpoint of Eq. (23). Then e belongs to one and only one of the following two cases:

(i) (NONOSC) Eq. (23) is non-oscillatory at e for all real $\lambda$. Correspondingly, e is called non-oscillatory.

(ii) $(O-N O)$ There exists a real number $\Lambda \geq 0$ such that Eq. (23) is oscillatory at $e$ for all $\lambda>\Lambda$ and non-oscillatory at e for all $\lambda<\Lambda$. Correspondingly, $e$ is called $O-N O$ with cutoff $\Lambda$. Equation (23) can be either oscillatory or non-oscillatory at $e$ for $\lambda=\Lambda>0$. It is always non-oscillatory for $\lambda=0$.

Proof. The proof follows from the results in Glazman [31, p. 41] and Fulton et al. $[28$, Theorem 1] specialized to the non-negative SL operator $\mathcal{A}$.

Based on the oscillatory/non-oscillatory classification of boundaries, the spectrum of the non-negative SL operator $\mathcal{A}$ is classified as follows. 


\section{Theorem 2 (Spectral Classification).}

(i) Spectral Category I. If both endpoints are NONOSC, then the spectrum is simple, non-negative and purely discrete.

(ii) Spectral Category II. If one of the endpoints is NONOSC and the other endpoint is $O-N O$ with cutoff $\Lambda \geq 0$, then the spectrum is simple and nonnegative, the essential spectrum is nonempty, $\sigma_{e}(\mathcal{A}) \subset[\Lambda, \infty)$, and $\Lambda$ is the lowest point of the essential spectrum. If the $S L$ equation is non-oscillatory at the $O-N O$ endpoint for $\lambda=\Lambda \geq 0$, then there is a finite set of simple eigenvalues in $[0, \Lambda]$ (it may be empty). If the $S L$ equation is oscillatory at the $O-N O$ endpoint for $\lambda=\Lambda>0$, then there is an infinite sequence of simple eigenvalues in $[0, \Lambda)$ clustering at $\Lambda$.

(iii) Spectral Category III. If $e_{1}$ is $O-N O$ with cutoff $\Lambda_{1} \geq 0$ and $e_{2}$ is $O-N O$ with cutoff $\Lambda_{2} \geq 0$, then the essential spectrum is nonempty, $\sigma_{e}(\mathcal{A}) \subset[\underline{\Lambda}, \infty)$, $\underline{\Lambda}:=\min \left\{\Lambda_{1}, \Lambda_{2}\right\}$, and $\underline{\Lambda}$ is the lowest point of the essential spectrum. The spectrum is simple (has multiplicity one) below $\bar{\Lambda}:=\max \left\{\Lambda_{1}, \Lambda_{2}\right\}$ and is not simple (has multiplicity two) above $\bar{\Lambda}$. If the $S L$ equation is non-oscillatory for $\lambda=\underline{\Lambda} \geq 0$, then there is a finite set of simple eigenvalues in $[0, \underline{\Lambda}]$ (it may be empty). If the $S L$ equation is oscillatory for $\lambda=\underline{\Lambda}>0$, then there is an infinite sequence of simple eigenvalues in $[0, \underline{\Lambda})$ clustering at $\underline{\Lambda}$.

Proof. The proof follows from the results of the spectral classification in Fulton et al. [28, Section 7] specialized to the non-negative SL operator $\mathcal{A}$.

As we have seen in Sec. 3, if there are no natural boundaries, the spectrum of the infinitesimal generator is purely discrete. Hence, regular, exit and entrance boundaries are always NONOSC. Natural boundaries can be either NONOSC or O-NO with cutoff $\Lambda \geq 0$.

For standard Brownian motion, $-\infty$ and $+\infty$ are both natural and oscillatory for all $\lambda>0$ and non-oscillatory for all $\lambda \leq 0$. For Bessel processes with linear drift (see Sec. 6.3),$+\infty$ is a natural boundary and is non-oscillatory for all real $\lambda$. Thus, a natural boundary can be either NONOSC or O-NO with some cutoff $\Lambda \geq 0$. A natural boundary that is O-NO with cutoff $\Lambda>0$ can be either oscillatory or nonoscillatory for $\lambda=\Lambda>0$. The Brownian motion killed at the constant rate $r>0$ provides an example where the cutoff value $\Lambda=r>0$ is non-oscillatory. The Bessel process on $(0, \infty)$ with constant negative drift and with the infinitesimal generator

$$
\frac{1}{2} \frac{d^{2}}{d x^{2}}+\left(\frac{\nu+1 / 2}{x}+\mu\right) \frac{d}{d x}
$$

with $\nu>-1 / 2$ and $\mu<0$ provides an example of a diffusion with oscillatory cutoff $\Lambda=\frac{\mu^{2}}{2}$ and an infinite sequence of eigenvalues in $[0, \Lambda)$ clustering at $\Lambda$ (see Sec. 6.4 ).

To determine when a natural boundary is NONOSC or O-NO with cutoff $\Lambda$, it is convenient to transform the SL Eq. (23) to the Liouville normal form. The 
following smoothness assumptions are sufficient to be able to perform the Liouville transformation.

Assumption 2. $a^{\prime}(x), a^{\prime \prime}(x)$ and $b^{\prime}(x)$ exist and are continuous on $\left(e_{1}, e_{2}\right)$.

We now transform the SL Eq. (23) to the Liouville normal form (see also Carr et al. [10] where similar transformations are used to obtain closed-form option pricing formulas by reducing the option pricing PDE to the heat equation). Fix some $x_{0} \in\left(e_{1}, e_{2}\right)$ and transform the independent and dependent variables as follows:

$$
y=\sqrt{2} \int_{x_{0}}^{x} \frac{d z}{a(z)}, v(y)=\frac{2^{\frac{1}{4}} u(x(y))}{\sqrt{a(x(y)) \mathfrak{s}(x(y))}},
$$

where $x=x(y)$ is the inverse of the Liouville transformation $y=y(x)$. Then the function $v(y)$ satisfies the SL equation in the Liouville normal form (the coefficient in front of the second derivative is constant and equal to (negative) one and the first-derivative term is absent; the SL equation in the Liouville normal form is called the one-dimensional Schrödinger equation):

$$
-v^{\prime \prime}+Q(y) v=\lambda v, y \in\left(y\left(e_{1}\right), y\left(e_{2}\right)\right),
$$

where the potential function $Q(y)$ is given by

$$
\begin{gathered}
Q(y)=\mathcal{V}(x(y)), \\
\mathcal{V}(x):=\frac{1}{8}\left(a^{\prime}(x)\right)^{2}-\frac{1}{4} a(x) a^{\prime \prime}(x)+\frac{b^{2}(x)}{2 a^{2}(x)}+\frac{1}{2} b^{\prime}(x)-\frac{b(x) a^{\prime}(x)}{a(x)}+r(x) .
\end{gathered}
$$

Due to Assumption 2 the function $\mathcal{V}(x)$ is continuous on $\left(e_{1}, e_{2}\right)$.

The oscillatory/non-oscillatory classification remains invariant under the Liouville transformation, i.e., Eq. (23) is non-oscillatory at $e \in\left\{e_{1}, e_{2}\right\}$ for a particular $\lambda$ if and only if Eq. (26) is non-oscillatory at $y(e)$ for that $\lambda$ [28, Lemma 2]. The oscillatory/non-oscillatory classification of Eq. (26) depends on the behavior of the potential function $Q$ near the endpoints. To formulate the classification result, we make the following additional assumption.

Assumption 3. Let $e \in\left\{e_{1}, e_{2}\right\}$ be a natural boundary. Then we assume that the limit $\lim _{x \rightarrow e} \mathcal{V}(x)$ exists (it is allowed to be infinite).

Theorem 3 (NONOSC/O-NO Classification of Natural Boundaries). Suppose Assumptions 1-3 hold and $e \in\left\{e_{1}, e_{2}\right\}$ is a natural boundary.

(i) If e is transformed into a finite endpoint by the Liouville transformation, i.e., $y(e)=\sqrt{2} \int_{x_{0}}^{e} \frac{d z}{a(z)}$ is finite, then e is NONOSC.

(ii) Suppose e is transformed into $-\infty$ or $+\infty$ by the Liouville transformation. If $\lim _{x \rightarrow e} \mathcal{V}(x)=+\infty$, then $e$ is NONOSC. If for some finite $\Lambda$

$$
\lim _{x \rightarrow e} \mathcal{V}(x)=\Lambda \text {, }
$$

then $e$ is $O-N O$ with cutoff $\Lambda$. Since the $S L$ operator $\mathcal{A}$ is non-negative, it follows that $\Lambda \geq 0$. If $\Lambda>0$ and $\lim _{x \rightarrow e} y^{2}(x)(\mathcal{V}(x)-\Lambda)>-1 / 4$, then $e$ is 
non-oscillatory for $\lambda=\Lambda>0$. If $\Lambda>0$ and $\lim _{x \rightarrow e} y^{2}(x)(\mathcal{V}(x)-\Lambda)<-1 / 4$, then $e$ is oscillatory for $\lambda=\Lambda>0$. If $\Lambda=0$, e is always non-oscillatory for $\lambda=\Lambda=0$.

Proof. Part (i) follows from Dunford and Schwartz [22], Corollary 38, p. 1464 and Theorem 23, p. 1414 specialized to the non-negative SL operator. Part (ii) follows from Dunford and Schwartz [22], Corollary 57, p. 1481 specialized to the non-negative SL operator.

Theorem 2 tells us that O-NO natural boundaries generate some non-empty essential spectrum above the cutoff, but it does not identify the essential spectrum. It is well known that when the potential function oscillates towards an infinite boundary, the essential spectrum above the cutoff may have a very complicated structure. In particular, it may consist of an infinite sequence of disjoint intervals separated by gaps. Furthermore, eigenvalues may be present in the gaps or embedded in the continuous spectrum (see [79, p. 143]) for some examples of possible behavior). Adding Assumption 3 along with the following assumption yields a drastic simplification of the essential spectrum.

Assumption 4. Suppose Assumptions 1-3 hold. If $e_{1}$ is a O-NO natural boundary with cutoff $\Lambda$, we assume that there exists such $c \in\left(e_{1}, e_{2}\right)$ that the function $\mathcal{V}(x)$ has bounded variation on $\left(e_{1}, c\right]$. If $e_{2}$ is a O-NO natural boundary with cutoff $\Lambda$, we assume that there exists such $c \in\left(e_{1}, e_{2}\right)$ that the function $\mathcal{V}(x)$ has bounded variation on $\left[c, e_{2}\right)$.

Theorem 4 (Essential Spectrum Generated by O-NO Natural Boundaries). Suppose Assumptions 1-4 hold.

(i) Spectral Category II. If one of the boundaries is NONOSC and the other boundary is $O-N O$ with cutoff $\Lambda \geq 0$, then the essential spectrum of $\mathcal{A}$ is $\sigma_{e}(\mathcal{A})=[\Lambda, \infty)$. Moreover, $\mathcal{A}$ has purely absolutely continuous spectrum in $(\Lambda, \infty)$.

(ii) Spectral Category III. If $e_{1}$ is $O-N O$ with cutoff $\Lambda_{1} \geq 0$ and $e_{2}$ is $O$-NO with cutoff $\Lambda_{2} \geq 0$, then the essential spectrum of $\mathcal{A}$ is $\sigma_{e}(\mathcal{A})=[\underline{\Lambda}, \infty), \underline{\Lambda}:=$ $\min \left\{\Lambda_{1}, \Lambda_{2}\right\}$. Moreover, $\mathcal{A}$ has purely absolutely continuous spectrum in $(\underline{\Lambda}, \infty)$. The part of the spectrum below $\bar{\Lambda}=\max \left\{\Lambda_{1}, \Lambda_{2}\right\}$ is simple (has multiplicity one). The part of the spectrum above $\bar{\Lambda}$ is not simple (has multiplicity two).

Proof. The proof follows from Dunford and Schwartz [22], p. 1448, Theorem 16(b) specialized to the non-negative SL operator and Weidmann [87], p. 233, Theorem 15.3.

Theorem 4 tells us that when Assumption 4 holds, the spectrum above the cutoff is purely absolutely continuous. Assumption 4 rules out parameters that oscillate towards O-NO natural boundaries. They are not particularly restrictive for financial 
applications (essentially all diffusion models in finance satisfy these assumptions), but yield a drastic simplification of the structure of the essential spectrum. Under Assumption 4, one can read off the structure of the spectrum directly from the behavior of $a, b$ and $r$ near the boundaries.

\section{The Simplified Form of the Spectral Representation for One-Dimensional Diffusions}

\subsection{Spectral Category I}

When there are no O-NO natural boundaries, the spectrum is simple, non-negative and purely discrete, and the spectral representation for the transition density and the spectral expansion for the value function take the form (20) and (21), respectively.

To described a general procedure to determine the eigenvalues and eigenfunctions, we need the following lemma.

\section{Lemma 1 (Solutions $\psi(x, \lambda)$ and $\phi(x, \lambda)$ at NONOSC Endpoints).}

(i) If $e_{1}$ is a NONOSC endpoint of the SL Eq. (23), then there exists a (unique up to a multiple independent of $x)$ non-trivial solution $\psi(x, \lambda)$ of the SL Eq. (23), which is square-integrable with $\mathfrak{m}$ in a neighborhood of $e_{1}$ for all $\lambda \in \mathbb{C}$, satisfies the appropriate boundary condition at $e_{1}$ (as described in Sec. 3) and such that $\psi(x, \lambda)$ and $\psi^{\prime}(x, \lambda)$ are continuous in $x$ and $\lambda$ in $I \times \mathbb{C}$ and entire in $\lambda$ for each fixed $x$.

(ii) If $e_{2}$ is a NONOSC endpoint of the SL Eq. (23), then there exists a (unique up to a multiple independent of $x)$ non-trivial solution $\phi(x, \lambda)$ of the SL Eq. (23), which is square-integrable with $\mathfrak{m}$ in a neighborhood of $e_{2}$ for all $\lambda \in \mathbb{C}$, satisfies the appropriate boundary condition at $e_{2}$ (as described in Sec. 3) and such that $\phi(x, \lambda)$ and $\phi^{\prime}(x, \lambda)$ are continuous in $x$ and $\lambda$ in $I \times \mathbb{C}$ and entire in $\lambda$ for each fixed $x$.

Proof. The proof follows from Dunford and Schwartz [22], p. 1472, Lemmas 41 and 42 and Fulton et al. [28], Theorems 16 and 18.

Let $\psi(x, \lambda)$ and $\phi(x, \lambda)$ be the solutions of Eq. (23) asserted to exist in Lemma 1. For any two functions $f, g \in A C_{\text {loc }}(I)$ define a Wronskian:

$$
[f, g](x):=f(x) \frac{g^{\prime}(x)}{\mathfrak{s}(x)}-g(x) \frac{f^{\prime}(x)}{\mathfrak{s}(x)} .
$$

From Eq. (23), the Wronskian of $\psi(x, \lambda)$ and $\phi(x, \lambda)$ is independent of $x$ and is entire in $\lambda$ (recall that $\psi$ and $\phi$ are entire in $\lambda$ ):

$$
[\psi(\cdot, \lambda), \phi(\cdot, \lambda)](x)=w(\lambda) .
$$

An eigenfunction $\varphi_{n}(x)$ is square-integrable with $\mathfrak{m}$ in a neighborhood of $e_{1}$ and satisfies the appropriate boundary condition at $e_{1}$, hence it must be equal to $\psi\left(x, \lambda_{n}\right)$ 
up to a non-zero constant multiple. But $\varphi_{n}(x)$ is also square-integrable with $\mathfrak{m}$ in a neighborhood of $e_{2}$ and satisfies the appropriate boundary condition at $e_{2}$, hence it must also be equal to $\phi\left(x, \lambda_{n}\right)$ up to a non-zero constant multiple. Thus, for $\lambda=\lambda_{n}$, $\psi\left(x, \lambda_{n}\right)$ and $\phi\left(x, \lambda_{n}\right)$ must be linearly dependent:

$$
\phi\left(x, \lambda_{n}\right)=A_{n} \psi\left(x, \lambda_{n}\right),
$$

for some non-zero constant $A_{n}$ and, hence, their Wronskian must vanish for $\lambda=\lambda_{n}$,

$$
w\left(\lambda_{n}\right)=0 .
$$

Conversely, let $\lambda_{n}$ be a zero of the Wronskian. Then $\psi\left(x, \lambda_{n}\right)$ and $\phi\left(x, \lambda_{n}\right)$ are linearly dependent, Eq. (32) holds for some non-zero $A_{n}$ and, hence, $\psi\left(x, \lambda_{n}\right)$ is a solution of Eq. (23) that is square-integrable with $\mathfrak{m}$ on $I$ and satisfies the appropriate boundary conditions at both end-points $e_{1}$ and $e_{2}$, i.e., $\psi\left(x, \lambda_{n}\right)$ is a (non-normalized) eigenfunction corresponding to the eigenvalue $\lambda_{n}$. Therefore, the eigenvalues $\left\{\lambda_{n}\right\}_{n=1}^{\infty}$ are zeros of $w(\lambda)$, and $\psi\left(x, \lambda_{n}\right)$ are the corresponding (nonnormalized) eigenfunctions. Since all eigenvalues of the SL operator $\mathcal{A}$ with $r(x) \geq 0$ are simple and non-negative, so are all zeros of the Wronskian $w(\lambda)$. Thus, practically, to determine the eigenvalues, find the solutions $\psi$ and $\phi$, compute their Wronskian and find its zeros. Finally, the normalized eigenfunctions are given by the following theorem.

Theorem 5. The normalized eigenfunctions can be taken in the form:

$$
\begin{aligned}
\varphi_{n}(x) & = \pm \frac{\psi\left(x, \lambda_{n}\right)}{\left\|\psi\left(\cdot, \lambda_{n}\right)\right\|}= \pm \frac{\phi\left(x, \lambda_{n}\right)}{\left\|\phi\left(\cdot, \lambda_{n}\right)\right\|}= \pm \sqrt{\frac{A_{n}}{w^{\prime}\left(\lambda_{n}\right)}} \psi\left(x, \lambda_{n}\right) \\
& = \pm \frac{\phi\left(x, \lambda_{n}\right)}{\sqrt{A_{n} w^{\prime}\left(\lambda_{n}\right)}},
\end{aligned}
$$

where $\left.w^{\prime}\left(\lambda_{n}\right) \equiv \frac{d w(\lambda)}{d \lambda}\right|_{\lambda=\lambda_{n}}$ and the $A_{n}$ are defined by Eq. (32).

Proof. Fix $x \in\left(e_{1}, e_{2}\right)$, take $\lambda>\lambda_{n}$ such that there are no eigenvalues in $\left(\lambda_{n}, \lambda\right]$ and observe that $(\mathcal{A} \psi)(x, \lambda)=\lambda \psi(x, \lambda)$ and $(\mathcal{A} \phi)(x, \lambda)=\lambda \phi(x, \lambda)$. We then have

$$
\begin{aligned}
(\lambda- & \left.\lambda_{n}\right) \int_{x}^{e_{2}} \psi\left(y, \lambda_{n}\right) \phi(y, \lambda) \mathfrak{m}(y) d y \\
& =\int_{x}^{e_{2}}\left[\psi\left(y, \lambda_{n}\right)(\mathcal{A} \phi)(y, \lambda)-(\mathcal{A} \psi)\left(y, \lambda_{n}\right) \phi(y, \lambda)\right] \mathfrak{m}(y) d y \\
& =-\int_{x}^{e_{2}} \frac{d\left[\psi\left(\cdot, \lambda_{n}\right), \phi(\cdot, \lambda)\right](y)}{d y} d y=\left[\psi\left(\cdot, \lambda_{n}\right), \phi(\cdot, \lambda)\right](x),
\end{aligned}
$$

where we have used the fact that $\lim _{x \uparrow e_{2}}\left[\psi\left(\cdot, \lambda_{n}\right), \phi(\cdot, \lambda)\right](x)=0$ since both $\psi\left(x, \lambda_{n}\right)$ and $\phi(x, \lambda)$ satisfy the appropriate boundary condition at $e_{2}$ and $\mathcal{A}$ is self-adjoint. 
Re-write the result as follows (recall that $\left.\left[\psi\left(\cdot, \lambda_{n}\right), \phi\left(\cdot, \lambda_{n}\right)\right](x)=0\right)$ :

$$
\begin{aligned}
\int_{x}^{e_{2}} & \psi\left(y, \lambda_{n}\right) \phi(y, \lambda) \mathfrak{m}(y) d y \\
= & {\left[\psi\left(\cdot, \lambda_{n}\right), \frac{\phi(\cdot, \lambda)-\phi\left(\cdot, \lambda_{n}\right)}{\lambda-\lambda_{n}}\right](x)+\left[\frac{\psi(\cdot, \lambda)-\psi\left(\cdot, \lambda_{n}\right)}{\lambda-\lambda_{n}}, \phi\left(\cdot, \lambda_{n}\right)\right](x) } \\
& -\left[\frac{\psi(\cdot, \lambda)-\psi\left(\cdot, \lambda_{n}\right)}{\lambda-\lambda_{n}}, \phi\left(\cdot, \lambda_{n}\right)\right](x),
\end{aligned}
$$

take the limit $\lambda \downarrow \lambda_{n}$ and $x \downarrow e_{1}$, observe that $\lim _{x \downarrow e_{1}}\left[\psi(\cdot, \lambda), \phi\left(\cdot, \lambda_{n}\right)\right](x)=0$ for any fixed $\lambda$ since both $\psi(x, \lambda)$ and $\phi\left(x, \lambda_{n}\right)$ satisfy the appropriate boundary condition at $e_{1}$ and $\mathcal{A}$ is self-adjoint, and recall Eq. (31) to obtain:

$$
\int_{e_{1}}^{e_{2}} \psi\left(y, \lambda_{n}\right) \phi\left(y, \lambda_{n}\right) \mathfrak{m}(y) d y=w^{\prime}\left(\lambda_{n}\right) .
$$

The result (34) immediately follows by recalling Eq. (32).

\subsection{Spectral Category II}

Suppose $e_{1}$ is NONOSC and $e_{2}$ is a O-NO natural boundary with cutoff $\Lambda \geq 0$ (the case of O-NO $e_{1}$ and NONOSC $e_{2}$ is treated similarly). The essential spectrum of $\mathcal{A}$ is $\sigma_{e}(\mathcal{A})=[\Lambda, \infty)$. Moreover, $\mathcal{A}$ has purely absolutely continuous spectrum in $(\Lambda, \infty)$. If $e_{2}$ is non-oscillatory for $\lambda=\Lambda \geq 0$, then there is a finite set of simple eigenvalues in $[0, \Lambda]$ (it may be empty). If $e_{2}$ is oscillatory for $\lambda=\Lambda>0$, then there is an infinite sequence of simple eigenvalues in $[0, \Lambda)$ clustering towards $\Lambda$. Accordingly, the spectral representation (12) for the transition density and the spectral expansion (17) for the value function simplify to:

$$
\begin{gathered}
p(t ; x, y)=\mathfrak{m}(y)\left(\sum_{n} e^{-\lambda_{n} t} \varphi_{n}(x) \varphi_{n}(y)+\int_{\Lambda}^{\infty} e^{-\lambda t} \psi(x, \lambda) \psi(y, \lambda) d \rho_{a c}(\lambda)\right), \\
t>0 \\
V(t, x)=\sum_{n} c_{n} e^{-\lambda_{n} t} \varphi_{n}(x)+\int_{\Lambda}^{\infty} e^{-\lambda t} F(\lambda) \psi(x, \lambda) d \rho_{a c}(\lambda), \quad x \in I, \quad t \geq 0, \\
c_{n}=\left(f, \varphi_{n}\right), F(\lambda)=\int_{I} f(y) \psi(y, \lambda) \mathfrak{m}(y) d y, \quad f \in L^{2}(I, \mathfrak{m}) .
\end{gathered}
$$

Here $\varphi_{n}(x)$ are eigenfunctions corresponding to the eigenvalues $\lambda_{n}$ (if any), $\psi(x, \lambda)$ is the solution of the SL Eq. (23) asserted to exist in Lemma 1, and $\rho_{a c}(\lambda)$ is the spectral function absolutely continuous on $(\Lambda, \infty)$ and normalized relative to $\psi(x, \lambda)$.

The eigenvalues and eigenfunctions below $\Lambda$ are determined as for the Spectral Category I. Lemma 1 can be extended to O-NO endpoints as follows. 


\section{Lemma 2 (Solutions $\psi(x, \lambda)$ and $\phi(x, \lambda)$ at O-NO Endpoints).}

(i) If $e_{1}$ is a O-NO endpoint of the $S L E q$. (23) with cutoff $\Lambda_{1} \geq 0$, then there exists a (unique up to a multiple independent of $x$ ) non-trivial solution $\psi(x, \lambda)$ of the SL Eq. (23), which is square-integrable with $\mathfrak{m}$ in a neighborhood of $e_{1}$ for all $\lambda \in S_{\Lambda_{1}}$, satisfies the appropriate boundary condition at $e_{1}$ (as described in Sec. 3) and such that $\psi(x, \lambda)$ and $\psi^{\prime}(x, \lambda)$ are continuous in $x$ and $\lambda$ in $I \times S_{\Lambda_{1}}$ and analytic in $\lambda$ in $S_{\Lambda_{1}}$ for each fixed $x$, where $S_{\Lambda_{1}}:=\left\{\lambda \in \mathbb{C}: \operatorname{Re}(\lambda)<\Lambda_{1}\right\}$.

(ii) If $e_{2}$ is a $O$-NO endpoint of the $S L E q$. (23) with cutoff $\Lambda_{2} \geq 0$, then there exists a (unique up to a multiple independent of $x$ ) non-trivial solution $\phi(x, \lambda)$ of the SL Eq. (23), which is square-integrable with $\mathfrak{m}$ in a neighborhood of $e_{2}$ for all $\lambda \in S_{\Lambda_{2}}$, satisfies the appropriate boundary condition at $e_{2}$ (as described in Sec. 3) and such that $\phi(x, \lambda)$ and $\phi^{\prime}(x, \lambda)$ are continuous in $x$ and $\lambda$ in $I \times S_{\Lambda_{2}}$ and analytic in $\lambda$ in $S_{\Lambda_{2}}$ for each fixed $x$, where $S_{\Lambda_{2}}:=\left\{\lambda \in \mathbb{C}: \operatorname{Re}(\lambda)<\Lambda_{2}\right\}$.

Proof. The proof follows from Dunford and Schwartz [22], p. 1472, Lemmas 41 and 42 and Fulton et al. [28], Theorem 19.

If $e_{1}$ is NONOSC and $e_{2}$ is O-NO with cutoff $\Lambda$, then the eigenvalues $\lambda$ can be found as zeros (if any) of the Wronskian $w(\lambda)$ of the two solutions $\psi(x, \lambda)$ and $\phi(x, \lambda)$ asserted to exist in Lemmas 1 and 3 in the half-plane $S_{\Lambda}$ (the two solutions and their Wronskian are analytic in this half-plane), and the corresponding normalized eigenfunctions are determined as in Lemma 2.

Since the absolutely continuous spectrum has multiplicity one, we are able to write down the continuous part of the spectral expansion in terms of just one spectral function in contrast to the general case that requires a $2 \times 2$ spectral matrix. There are two approaches to obtain the spectral function, the complex variable approach of Titchmarsh [84] and the real variable approach of Levitan [54] and Levinson [53] (see also $[11,55,69]$ ). Here we briefly outline the real variable approach. Pick some $b \in\left(e_{1}, e_{2}\right)$ and kill the process $\hat{X}$ at the first hitting time of $b, \mathcal{T}_{b}:=\inf \left\{t \geq 0: \hat{X}_{t}=b\right\}$. The process killed at $b, \hat{X}_{t}^{b}:=\hat{X}_{t}(\partial)$ if $t<$ $\mathcal{T}_{b}\left(t \geq \mathcal{T}_{b}\right)$, has the state space $I^{b} \cup\{\partial\}$ with NONOSC boundary $e_{1}$ and regular killing boundary $b$. If $e_{1}$ is a regular reflecting boundary, $I^{b}=\left[e_{1}, b\right)$. Otherwise, $I^{b}=\left(e_{1}, b\right)$. The diffusion $\hat{X}^{b}$ generates a strongly continuous semigroup of selfadjoint contractions $\left\{\mathcal{P}_{t}^{b}, t \geq 0\right\}$ in the Hilbert space $L^{2}\left(I^{b}, \mathfrak{m}\right)$ with the inner product $(f, g)^{b}=\int_{I^{b}} f(x) g(x) \mathfrak{m}(x) d x,\left(\mathcal{P}_{t}^{b} f\right)(x):=\mathbb{E}_{x}\left[f\left(\hat{X}_{t}^{b}\right)\right]=\mathbb{E}_{x}\left[\mathbf{1}_{\left\{t<\mathcal{T}_{b}\right\}} f\left(\hat{X}_{t}\right)\right]$, $f \in L^{2}\left(I^{b}, \mathfrak{m}\right), x \in I^{b}, t \geq 0$. Since both boundaries are NONOSC, we are in the Spectral Category I. The eigenvalues $\left\{\lambda_{n, b}\right\}_{n=1}^{\infty}$ are the (simple, positive) roots of the equation

$$
\psi(b, \lambda)=0,
$$


and the spectral representation for the transition density of $\hat{X}^{b}$ can be written in the form

$$
p^{b}(t ; x, y)=\mathfrak{m}(y) \sum_{n=1}^{\infty} e^{-\lambda_{n, b} t} \frac{\psi\left(x, \lambda_{n, b}\right) \psi\left(y, \lambda_{n, b}\right)}{\left\|\psi\left(\cdot, \lambda_{n, b}\right)\right\|_{b}^{2}}
$$

where

$$
\left\|\psi\left(\cdot, \lambda_{n, b}\right)\right\|_{b}^{2}=\left(\psi\left(\cdot, \lambda_{n, b}\right), \psi\left(\cdot, \lambda_{n, b}\right)\right)^{b}
$$

and we explicitly show dependence on $b$.

Introduce a nondecreasing right-continuous jump function (the spectral function of the problem on $I^{b}$ ):

$$
\rho^{b}(\lambda):=\sum_{n=1}^{\infty} \frac{1}{\left\|\psi\left(\cdot, \lambda_{n, b}\right)\right\|_{b}^{2}} \mathbf{1}_{\left\{\lambda_{n, b} \leq \lambda\right\}},
$$

where $\mathbf{1}_{\left\{\lambda_{n, b} \leq \lambda\right\}}=1(0)$ if $\lambda_{n, b} \leq \lambda\left(\lambda_{n, b}>\lambda\right)$. It jumps by $\left\|\psi\left(\cdot, \lambda_{n, b}\right)\right\|^{-2}$ at an eigenvalue $\lambda=\lambda_{n, b}$. The spectral representation (39) can now be re-written in the equivalent form as a Stieltjes integral:

$$
p^{b}(t ; x, y)=\mathfrak{m}(y) \int_{[0, \infty)} e^{-\lambda t} \psi(x, \lambda) \psi(y, \lambda) d \rho^{b}(\lambda) .
$$

The limit $\lim _{b \uparrow e_{2}} \rho^{b}(\lambda)=\rho(\lambda)$ produces a nondecreasing right-continuous function - the spectral function of the original problem on $I$ (e.g., [55, Chapter 2]). The spectral representation for the original problem on $I$ can be derived from (41) by letting $b \uparrow e_{2}$ :

$$
p(t ; x, y)=\mathfrak{m}(y) \int_{[0, \infty)} e^{-\lambda t} \psi(x, \lambda) \psi(y, \lambda) d \rho(\lambda) .
$$

Since the problem on $I$ has purely absolutely continuous spectrum above $\Lambda$ plus possibly some eigenvalues $\left\{\lambda_{n}\right\}$ in $[0, \Lambda]$, the spectral function can be written in the form:

$$
\rho(\lambda)=\sum_{n} \frac{1}{\left\|\psi\left(\cdot, \lambda_{n}\right)\right\|^{2}} \mathbf{1}_{\left\{\lambda_{n} \leq \lambda\right\}}+\rho_{a c}(\lambda) \mathbf{1}_{\{\Lambda \leq \lambda\}},
$$

where the sum is over the eigenvalues (if any) and $\rho_{a c}(\lambda)$ is the function absolutely continuous for $\lambda>\Lambda$ and vanishing for $\lambda<\Lambda$. Substituting (43) into (42), we recover the spectral expansion (35). Roughly speaking, the discrete spectrum above $\Lambda$ of the problem on $I^{b}$ merges into the continuous spectrum of the problem on $I$ in the limit $b \uparrow e_{2}$ (as $b$ increases, the eigenvalues are distributed denser and denser and in the limit merge to form the continuous spectrum). 


\subsection{Spectral Category III}

Suppose $e_{1}$ is an O-NO natural boundary with cutoff $\Lambda_{1} \geq 0$ and $e_{2}$ is a O-NO natural boundary with cutoff $\Lambda_{2} \geq 0$. To be specific, suppose $\Lambda_{1}<\Lambda_{2}$. The essential spectrum of $\mathcal{A}$ is $\sigma_{e}(\mathcal{A})=\left[\Lambda_{1}, \infty\right)$. Moreover, $\mathcal{A}$ has purely absolutely continuous spectrum in $\left(\Lambda_{1}, \infty\right)$. The part of the spectrum below $\Lambda_{2}$ is simple (has multiplicity one). The part of the spectrum above $\Lambda_{2}$ is not simple (has multiplicity two). If the SL Eq. (23) is non-oscillatory for $\lambda=\Lambda_{1}$, there is a finite set of simple eigenvalues in $\left[0, \Lambda_{1}\right]$ (it may be empty). If the SL Eq. (23) is oscillatory for $\lambda=\Lambda_{1}>0$, then there is an infinite sequence of simple eigenvalues in $\left[0, \Lambda_{1}\right)$ clustering towards $\Lambda_{1}$. The spectral representation (12) for the transition density and the spectral expansion (17) for the value function take the form:

$$
\begin{gathered}
p(t ; x, y)=\mathfrak{m}(y) \sum_{n} e^{-\lambda_{n} t} \varphi_{n}(x) \varphi_{n}(y)+\mathfrak{m}(y) \int_{\Lambda_{1}}^{\Lambda_{2}} e^{-\lambda t} \phi(x, \lambda) \phi(y, \lambda) d \rho_{a c}(\lambda) \\
+\mathfrak{m}(y) \int_{\Lambda_{2}}^{\infty} e^{-\lambda t} \sum_{i, j=1}^{2} u_{i}(x, \lambda) u_{j}(y, \lambda) d \rho_{a c, i j}(\lambda), \quad x, y \in\left(e_{1}, e_{2}\right), \quad t>0, \\
V(t, x)=\sum_{n} c_{n} e^{-\lambda_{n} t} \varphi_{n}(x)+\int_{\Lambda_{1}}^{\Lambda_{2}} e^{-\lambda t} F(\lambda) \phi(x, \lambda) d \rho_{a c}(\lambda) \\
+\int_{\Lambda_{2}}^{\infty} e^{-\lambda t} \sum_{i, j=1}^{2} u_{i}(x, \lambda) F_{j}(\lambda) d \rho_{a c, i j}(\lambda), \quad x \in\left(e_{1}, e_{2}\right), \quad t \geq 0, \quad f \in L^{2}(I, \mathfrak{m}), \\
c_{n}=\left(f, \varphi_{n}\right), \quad F(\lambda)=\int_{I} f(y) \phi(y, \lambda) \mathfrak{m}(y) d y, \quad F_{i}(\lambda)=\int_{I} f(y) u_{i}(y, \lambda) \mathfrak{m}(y) d y,
\end{gathered}
$$

where $\rho_{a c}(\lambda)$ is the spectral function absolutely continuous on the simple segment of the spectrum between $\Lambda_{1}$ and $\Lambda_{2}, \rho_{a c, i j}(\lambda)$ is the $2 \times 2$ spectral matrix absolutely continuous on the multiplicity-two segment of the spectrum above $\Lambda_{2}, \lambda_{n}$ are eigenvalues in $\left[0, \Lambda_{1}\right]$ (if any) and $\varphi_{n}(x)$ are the corresponding eigenfunctions, $\phi(x, \lambda)$ is the solution asserted to exist in Lemma 2 , and $u_{i}(x, \lambda)$ are the solutions of the initial value problem (13)-(15) with the obvious substitution $\lambda \rightarrow-\lambda$. The modifications necessary for the cases $\Lambda_{1}>\Lambda_{2}$ and $\Lambda_{1}=\Lambda_{2}$ are obvious.

The eigenvalues and eigenfunctions and the spectral function $\rho_{a c}(\lambda)$ are determined as for the Spectral Category II. The $2 \times 2$ spectral matrix $\rho_{a c, i j}(\lambda)$ is determined as follows (we follow McKean's [69] original derivation). Pick some $e_{1}<a<b<e_{2}$ and kill the process $\hat{X}$ at the first exit time from $(a, b)$, $\mathcal{T}_{(a, b)}:=\inf \left\{t \geq 0: \hat{X}_{t} \notin(a, b)\right\}$. The process $\hat{X}^{(a, b)}$ killed at $a$ and $b$, $\hat{X}_{t}^{(a, b)}:=\hat{X}_{t}(\partial)$ if $t<\mathcal{T}_{(a, b)}\left(t \geq \mathcal{T}_{(a, b)}\right)$, has the state space $(a, b)$ with the regular killing boundaries $a$ and $b$ and generates a strongly continuous semigroup of self-adjoint contractions $\left\{\mathcal{P}_{t}^{(a, b)}, t \geq 0\right\}$ in $L^{2}((a, b), \mathfrak{m})$ with the inner product $(f, g)^{(a, b)}=\int_{a}^{b} f(x) g(x) \mathfrak{m}(x) d x,\left(\mathcal{P}_{t}^{(a, b)} f\right)(x):=\mathbb{E}_{x}\left[f\left(\hat{X}_{t}^{(a, b)}\right)\right]=$ 
$\mathbb{E}_{x}\left[\mathbf{1}_{\left\{t<\mathcal{T}_{(a, b)}\right\}} f\left(\hat{X}_{t}\right)\right], f \in L^{2}((a, b), \mathfrak{m}), x \in(a, b), t \geq 0$. Consider the spectral representation for this process with two regular killing boundaries:

$$
p^{(a, b)}(t ; x, y)=\mathfrak{m}(y) \sum_{n=1}^{\infty} e^{-\lambda_{n}^{(a, b)} t} \varphi_{n}^{(a, b)}(x) \varphi_{n}^{(a, b)}(y)
$$

where we explicitly show dependence on $a$ and $b$. The eigenfunction $\varphi_{n}^{(a, b)}(x)$ can be expressed in terms of $u_{i}(x, \lambda)$ with $x_{0} \in(a, b)$ as follows (to lighten notation we omit the superscript $(a, b))$ :

$$
\varphi_{n}(x)=\varphi_{n}\left(x_{0}\right) u_{1}\left(x, \lambda_{n}\right)+\frac{\varphi_{n}^{\prime}\left(x_{0}\right)}{\mathfrak{s}\left(x_{0}\right)} u_{2}\left(x, \lambda_{n}\right) .
$$

Introduce the following right-continuous jump functions:

$$
\begin{gathered}
\rho_{11}^{(a, b)}(\lambda):=\sum_{n=1}^{\infty} \varphi_{n}^{2}\left(x_{0}\right) \mathbf{1}_{\left\{\lambda_{n} \leq \lambda\right\}}, \rho_{22}^{(a, b)}(\lambda):=\sum_{n=1}^{\infty}\left(\frac{\varphi_{n}^{\prime}\left(x_{0}\right)}{\mathfrak{s}\left(x_{0}\right)}\right)^{2} \mathbf{1}_{\left\{\lambda_{n} \leq \lambda\right\}}, \\
\rho_{12}^{(a, b)}(\lambda)=\rho_{21}^{(a, b)}(\lambda):=\sum_{n=1}^{\infty} \frac{\varphi_{n}\left(x_{0}\right) \varphi_{n}^{\prime}\left(x_{0}\right)}{\mathfrak{s}\left(x_{0}\right)} \mathbf{1}_{\left\{\lambda_{n} \leq \lambda\right\}} .
\end{gathered}
$$

Now the spectral representation (47) can be re-written in the Stieltjes integral form:

$$
p^{(a, b)}(t ; x, y)=\mathfrak{m}(y) \int_{[0, \infty)} e^{-\lambda t} \sum_{i, j=1}^{2} u_{i}(x, \lambda) u_{j}(y, \lambda) d \rho_{i j}^{(a, b)}(\lambda) .
$$

In the limit $a \downarrow e_{1}$ and $b \uparrow e_{2}$ this spectral representation converges to the spectral representation (12) for the original problem on $\left(e_{1}, e_{2}\right)$ with natural boundaries at $e_{1}$ and $e_{2}$.

\section{Analytically Tractable Diffusion Models}

In order to determine the spectral representation explicitly, one needs explicit solutions of the SL equation. The Liouville transformation reduces the SL equation to the Schrödinger equation with potential (27-28). If analytical solutions are available for the Schrödinger equation, inverting the Liouville transformation yields analytical solutions for the original SL equation, and the spectral representation can be constructed explicitly according to the recipe of Sec. 5. In this Section we survey some analytically tractable models whose spectral representations can be expressed in terms of classical special functions. ${ }^{\text {a }}$

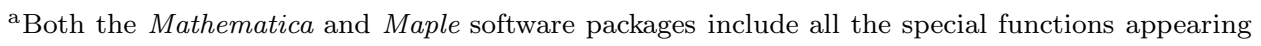
in this section as built-in functions. 


\subsection{Double barrier options in the Black-Scholes model}

Assume that under the risk-neutral probability measure the underlying asset price follows a geometric Brownian motion with the initial price $S_{0}=x$, constant volatility $\sigma>0$, constant risk-free rate $r \geq 0$ and constant dividend yield $q \geq 0$. In this case the infinitesimal parameters are:

$$
a(x)=\sigma x, \quad b(x)=(r-q) x,
$$

and the speed density is:

$$
\mathfrak{m}(x)=\frac{2}{\sigma^{2}} x^{\frac{2 \nu}{\sigma}-1},
$$

where

$$
\nu:=\frac{1}{\sigma}\left(r-q-\frac{\sigma^{2}}{2}\right) .
$$

Consider a double-barrier call option with the strike price $K$, expiration date $T$, and two knock-out barriers $L$ and $U, 0<L<K<U$. The knock-out provision renders the option worthless as soon as the underlying price leaves the price range $(L, U)$ (it is assumed that $S_{0} \in(L, U)$ ). References on double-barrier options include Kunitomo and Ikeda [50], Geman and Yor [30], Pelsser [75], Lipton [66], Davydov and Linetsky [17, 19] and Lipton and McGhee [68] (see also Linetsky [59] and Davydov and Linetsky [18] for some user-friendly alternatives to barrier options). The double barrier call payoff is $\mathbf{1}_{\left\{\mathcal{T}_{(L, U)}>T\right\}}\left(S_{T}-K\right)^{+}$, where $\mathcal{T}_{(L, U)}=\inf \{t \geq 0$ : $\left.S_{t} \notin(L, U)\right\}$ is the first exit time from the range $(L, U)$. The SL problem in this case is

$$
-\frac{1}{2} \sigma^{2} x^{2} u^{\prime \prime}-(r-q) x u^{\prime}+r u=\lambda u, \quad u(L)=u(U)=0 .
$$

The Liouville transformation

$$
y=\frac{\sqrt{2}}{\sigma} \ln \left(\frac{x}{L}\right), \quad u(x)=2^{-\frac{1}{4}} \sigma^{\frac{1}{2}} x^{-\frac{\nu}{\sigma}} v(y(x))
$$

reduces the SL equation to the Schrödinger equation with constant potential:

$$
-v^{\prime \prime}+Q v=\lambda v, \quad Q=r+\frac{\nu^{2}}{2}, \quad v(0)=0, \quad v(B)=0, \quad B=\frac{\sqrt{2}}{\sigma} \ln \left(\frac{U}{L}\right) .
$$

Both boundaries are regular killing and, hence, we are in the Spectral Category I. The solutions of Lemma 1 entire in $\lambda$ are:

$$
\psi(y, \lambda)=\frac{\sin (y \sqrt{\lambda-Q})}{\sqrt{\lambda-Q}}, \quad \phi(y, \lambda)=\frac{\sin (\sqrt{\lambda-Q}(B-y))}{\sqrt{\lambda-Q}} .
$$

The Wronskian

$$
w(\lambda)=-\frac{\sin (B \sqrt{\lambda-Q})}{\sqrt{\lambda-Q}},
$$


is entire in $\lambda$ with simple positive zeros:

$$
\lambda_{n}=Q+\frac{n^{2} \pi^{2}}{B^{2}}, \quad n=1,2, \ldots .
$$

At $\lambda=\lambda_{n}$ the two solutions become linearly dependent, $\phi\left(x, \lambda_{n}\right)=$ $(-1)^{n+1} \psi\left(x, \lambda_{n}\right)$, the Wronskian derivative at $\lambda=\lambda_{n}$ is $w^{\prime}\left(\lambda_{n}\right)=(-1)^{n+1} \frac{B^{3}}{2 n^{2} \pi^{2}}$, and the eigenfunctions (34) are given by

$$
\varphi_{n}(y)=\sqrt{\frac{2}{B}} \sin \left(\frac{n \pi y}{B}\right), \quad n=1,2, \ldots .
$$

Inverting the Liouville transformation yields the eigenfunctions and eigenvalues of the original problem (50):

$$
\begin{aligned}
\varphi_{n}(x) & =\frac{\sigma x^{-\frac{\nu}{\sigma}}}{\sqrt{\ln (U / L)}} \sin \left(\frac{\pi n \ln (x / L)}{\ln (U / L)}\right), \\
\lambda_{n} & =r+\frac{\nu^{2}}{2}+\frac{\sigma^{2} \pi^{2} n^{2}}{2 \ln ^{2}(U / L)}, \quad n=1,2, \ldots .
\end{aligned}
$$

The call option value function is given by

$$
C(t, x)=\sum_{n=1}^{\infty} c_{n} e^{-\lambda_{n} t} \varphi_{n}(x)
$$

with the expansion coefficients:

$$
c_{n}=\left((\cdot-K)^{+}, \varphi_{n}\right)=\frac{L^{\frac{\nu}{\sigma}}}{\sqrt{\ln (U / L)}}\left[L \psi_{n}(\nu+\sigma)-K \psi_{n}(\nu)\right],
$$

where

$$
\psi_{n}(a):=\frac{2}{\omega_{n}^{2}+a^{2}}\left[e^{a k}\left(\omega_{n} \cos \left(\omega_{n} k\right)-a \sin \left(\omega_{n} k\right)\right)-(-1)^{n} \omega_{n} e^{a u}\right],
$$

and

$$
\omega_{n}:=\frac{n \pi}{u}, \quad k:=\frac{1}{\sigma} \ln \left(\frac{K}{L}\right), \quad u:=\frac{1}{\sigma} \ln \left(\frac{U}{L}\right) .
$$

A practically important observation is that the eigenvalues increase as $n^{2}$ to facilitate fast convergence of the eigenfunction expansion. The longer the time to expiration, the faster the eigenfunction expansion converges. The option delta and gamma can be obtained by differentiating the eigenfunction expansion term-byterm:

$$
\begin{aligned}
& \Delta(t, x)=C_{x}(t, x)=\sum_{n=1}^{\infty} c_{n} e^{-\lambda_{n} t} \varphi_{n}^{\prime}(x), \\
& \Gamma(t, x)=C_{x x}(t, x)=\sum_{n=1}^{\infty} c_{n} e^{-\lambda_{n} t} \varphi_{n}^{\prime \prime}(x) .
\end{aligned}
$$


Table 1. Convergence of spectral expansions for double-barrier call prices, deltas and gammas with $T=1 / 12$ (one month) and one year. The number in the $N$ column indicates how many terms are included in the truncated expansion (e.g., $N=2$ means that the first two terms with $n=1$ and $n=2$ are included in the expansions (51-52)). Parameters: $S_{0}=K=100$, $L=80, U=130, r=0.1, q=0, \sigma=0.25$.

\begin{tabular}{cccc}
\hline & Price & Delta & Gamma \\
\hline$N$ & \multicolumn{2}{c}{ Double-Barrier Call $T=1 / 12$ Years } \\
\hline 1 & 7.65069 & -0.020999 & -0.028245 \\
2 & 5.78096 & 0.932450 & 0.032490 \\
3 & 2.60902 & 0.720920 & 0.143401 \\
4 & 3.11276 & 0.480955 & 0.102269 \\
5 & 3.35713 & 0.536457 & 0.078607 \\
6 & 3.29822 & 0.561105 & 0.088242 \\
7 & 3.28703 & 0.554994 & 0.090335 \\
8 & 3.28941 & 0.554203 & 0.089673 \\
9 & 3.28958 & 0.554412 & 0.089623 \\
10 & 3.28953 & 0.554422 & 0.089643 \\
11 & 3.28953 & 0.554419 & 0.089643 \\
\hline$N$ & Double-Barrier Call $T=1$ Years \\
\hline 1 & 2.03207 & -0.0055774 & -0.0075021 \\
2 & 2.01848 & 0.0013543 & -0.0070606 \\
3 & 2.01842 & 0.0013505 & -0.0070586 \\
4 & 2.01842 & 0.0013505 & -0.0070586 \\
\hline
\end{tabular}

To illustrate, Table 1 shows convergence of spectral expansions for double-barrier call option prices, deltas and gammas with $T=1 / 12,1 / 2$ and one year to expiration and $S_{0}=K=100, L=80, U=130, r=0.1, q=0, \sigma=0.25$. For one-year options, the first three terms are enough to converge to five significant digits. For one-month options $(T=1 / 12)$, the first ten terms are required to achieve this level of accuracy. There is no loss of accuracy in computing delta and gamma. This is in contrast to numerical option pricing methods such as lattices, numerical PDE schemes and simulation. Figure 1 plots call prices for $T=0.25,0.5$ and one year to expiration as functions of the underlying asset price $S_{0}$.

Remark on rebates. In the example above, as well as in other examples throughout this paper, we have not discussed a possibility of a rebate to be paid when the barrier is hit. If a cash rebate $R$ is paid, then the boundary condition is $u(B)=R$. We can separate the claim into two parts: the claim without rebate with the payoff at maturity if the barrier is not hit plus a rebate claim that pays the rebate amount if the barrier is hit prior to maturity. To calculate the value function of the rebate claim, one can use the Laplace transform technique. Similarly, when pricing a vanilla put option on a process that can attain the origin (bankruptcy state), one can split the put payoff into the two claims: put payoff at maturity, 


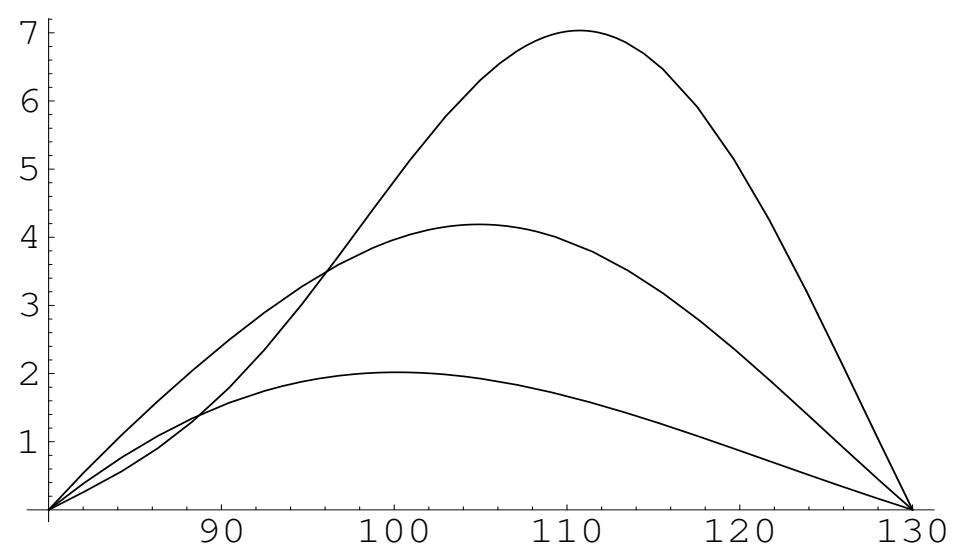

Fig. 1. Double-barrier call option price as a function of the underlying asset price for $T=0.25,0.5$ and one year to expiration (the longer the time to expiration, the lower the option price in the region near the upper barrier $U$ due to the increased probability of knockout prior to expiration). Parameters: $K=100, r=0.1, q=0, \sigma=0.25, L=80, U=130$.

given the origin is not hit prior to maturity plus the so-called "bankruptcy claim" - getting paid the strike price at maturity if the origin is hit. The value of the bankrupcy claim is equal to the value the probability of hitting the origin times the strike. See Lewis [57] for the calculation of the bankruptcy claim in Merton's cash dividend model, Davydov and Linetsky [17] for the calculation of the bankruptcy claim in the CEV model, and Davydov and Linetsky [19] for the pricing of barrier options with rebates in the CEV model.

\subsection{Harmonic oscillator and Ornstein-Uhlenbeck process}

\subsubsection{Ornstein-Uhlenbeck process}

Our next example is the Ornstein-Uhlenbeck process with the infinitesimal parameters

$$
a(x)=\sigma>0, \quad b(x)=\kappa(\theta-x), \quad \kappa>0 .
$$

The Liouville transformation (25) with $x_{0}=\theta$ reduces the SL Eq. (23) to the Schrödinger equation with the quadratic harmonic oscillator potential [73, p. 1641]:

$$
Q(y)=\frac{\kappa^{2}}{4} y^{2}-\frac{\kappa}{2}
$$

If the OU process is considered on the real line, both $-\infty$ and $+\infty$ are NONOSC natural boundaries $(Q(y) \rightarrow+\infty$ as $x \rightarrow \pm \infty)$ and the spectrum is purely discrete. The solutions $\psi(x, \lambda)$ and $\phi(x, \lambda)$ are expressed in terms of the WeberHermite parabolic cylinder functions. At an eigenvalue, $\lambda=\lambda_{n}$, the Weber-Hermite functions degenerate into Hermite polynomials $H_{n}(x)$ and the eigenfunctions are 
expressed in terms of the latter (e.g., [88] and [46, p. 333]; for notational convenience here we label the eigenvalues and eigenfunctions starting from $n=0$ ):

$$
\begin{aligned}
\lambda_{n} & =\kappa n, \quad \varphi_{n}(x)=\mathcal{N}_{n} H_{n}(\xi), \quad n=0,1, \ldots, \\
\xi & :=\frac{\sqrt{\kappa}}{\sigma}(x-\theta), \quad \mathcal{N}_{n}^{2}=\frac{\sigma \sqrt{\kappa}}{2^{n+1} n ! \sqrt{\pi}} .
\end{aligned}
$$

If the OU process is considered on some interval $I \subset \mathbb{R}$ with killing or reflection at finite boundaries, then the eigenfunctions are expressed in terms of the full WeberHermite functions.

\subsubsection{Vasicek, quadratic and Black's interest rate models}

If we take $r(x)=x$, we obtain the spectral representation for the state-price density of the Vasicek [85] interest rate model. In the Vasicek model the short rate $r$ can get negative. Consequently, the pricing semigroup is not, in general, a contraction semigroup. This leads to serious economic problems in the Vasicek model (see [34]). If we take $r(x)=x^{2}$, we obtain the spectral representation for the state-price density of a non-negative interest rate model where the short rate is a square of the Vasicek process (this quadratic model was proposed in [4]; see also [44]). For further details, explicit formulas and relevant references see Gorovoi and Linetsky [34], where a non-negative interest rate model with $r(x)=x^{+} \equiv \max \{x, 0\}$ is solved analytically and calibrated to empirical data.

\subsection{Radial harmonic oscillator, Bessel processes with linear drift and CIR, 3/2 and CEV models}

6.3.1. Bessel processes with linear drift

Consider a diffusion with infinitesimal parameters

$$
\begin{aligned}
& a(x)=1, \quad b(x)=\frac{\nu+\frac{1}{2}}{x}+c x, \quad r(x)=\frac{\alpha^{2}}{2 x^{2}}+\frac{\beta^{2}}{2} x^{2}, \\
& x \in(0, \infty), \quad \nu, c \in \mathbb{R}, \quad \alpha, \beta \geq 0 .
\end{aligned}
$$

The Liouville transform (25) with $x_{0}=0$ reduces the SL Eq. (23) to the Schrödinger equation with the radial harmonic oscillator potential [73, p. 1661]:

$$
Q(y)=\frac{\nu^{2}+\alpha^{2}-\frac{1}{4}}{y^{2}}+c(\nu+1)+\frac{c^{2}+\beta^{2}}{4} y^{2}, \quad y \in(0, \infty) .
$$

First consider the case with $\alpha=\beta=0$. For $\nu=d / 2-1$ with some integer $d \geq 2$ this process arises as a radial part of the $d$-dimensional Ornstein-Uhlenbeck process with the infinitesimal generator

$$
\frac{1}{2} \Delta+c x \cdot \nabla
$$


where $\Delta$ is the standard $d$-dimensional Laplacian (infinitesimal generator of $d$ dimensional Brownian motion). This process is called a radial Ornstein-Uhlenbeck process $[32,78,82]$. Here we consider the process with $\nu, c \in \mathbb{R}$ and call it a Bessel process with linear drift. The boundary classification at the origin is independent of the drift parameter $c$. For all $c \in \mathbb{R}, 0$ is entrance for $\nu \geq 0$, regular for $-1<\nu<0$ (both killing and reflecting boundary conditions arise in finance applications) and exit for $\nu \leq-1$. For $c \neq 0$ and all $\nu \in \mathbb{R}, Q(y) \rightarrow \infty$ as $y \rightarrow+\infty$ and $+\infty$ is NONOSC natural. Thus, the spectrum is purely discrete (Spectral Category I). The solutions $\psi(x, \lambda)$ and $\phi(x, \lambda)$ are expressed in terms of the Whittaker functions (or, equivalently, in terms of the Kummer and Tricomi confluent hypergeometric functions). At an eigenvalue, $\lambda=\lambda_{n}$, the Whittaker functions degenerate into generalized Laguerre polynomials $L_{n}^{(\alpha)}(x)$, and the eigenfunctions are expressed in terms of the latter. If the process is considered on an interval with killing or reflection at finite boundaries, then the eigenfunctions are expressed in terms of the full Whittaker functions.

A practically important property of this process is that if we kill the process at the rate $r(x)=\frac{\alpha^{2}}{2} x^{-2}+\frac{\beta^{2}}{2} x^{2}$ with some $\alpha+\beta>0$, the killed process $\hat{X}$ is just as analytically tractable as the process $X$ with $\alpha=\beta=0$. Indeed, both processes lead to the same potential function (56); only the coefficients in front of $y^{-2}$ and $y^{2}$ need to be adjusted when we add the killing (if $\alpha>0$, then 0 is a NONOSC natural boundary for the process $\hat{X}$ with killing for all $\nu \in \mathbb{R}$ ).

For $c=0$ and $\alpha=\beta=0$, the process reduces to the standard Bessel process of order $\nu, Q(y) \rightarrow 0$ as $y \rightarrow+\infty$, and $+\infty$ is an O-NO natural boundary with cutoff $\Lambda=0$. Zero is not an eigenvalue and we have a simple and purely absolutely continuous spectrum $\sigma(\mathcal{A})=\sigma_{a c}(\mathcal{A})=[0, \infty)$ (Spectral Category II). The solution $\psi(x, \lambda)$ in Eq. (36) is expressed in terms of Bessel functions and the spectral representation (36) has an integral form (e.g., [46, p. 338 for $\nu=d / 2-1]$ ).

\subsubsection{The CIR model}

Let $\left\{X_{t}, t \geq 0\right\}$ be a Bessel process with $\nu \geq 0$ and with linear drift with $c<0$ and $\alpha=\beta=0$. For $\sigma>0$, the squared process $\left\{\frac{\sigma^{2}}{4} X_{t}^{2}, t \geq 0\right\}$ is a Feller's (1951) square-root diffusion on $(0, \infty)$ with the infinitesimal parameters (see also [88]):

$$
a(x)=\sigma \sqrt{x}, \quad b(x)=\kappa(\theta-x), \text { where } \theta:=-\frac{\sigma^{2}}{4 c}(\nu+1)>0, \quad \kappa:=-2 c>0 .
$$

This diffusion is known in finance as the CIR process (Cox, Ingersoll and Ross [14]) and is widely used as a model of interest rates (the CIR term structure model), stochastic volatility ([39]) and credit spreads. The spectrum is purely discrete and the spectral representation for the CIR transition density is immediately obtained from the spectral representation for the transition density of the Bessel process with 
linear drift. The eigenvalues and eigenfunctions are:

$$
\begin{gathered}
\lambda_{n}=\kappa n, \quad \varphi_{n}(x)=\mathcal{N}_{n} L_{n}^{(\alpha)}(\xi), \quad n=0,1, \ldots, \quad \xi:=\frac{2 \kappa x}{\sigma^{2}}, \quad \alpha:=\frac{2 \theta \kappa}{\sigma^{2}}-1, \\
\mathcal{N}_{n}^{2}=\left(\frac{2 \kappa}{\sigma^{2}}\right)^{\alpha} \frac{n ! \kappa}{\Gamma(n+\alpha+1)} .
\end{gathered}
$$

The state-price density of the CIR term structure model can be interpreted as the transition density for the CIR process killed at the rate $r(x)=x$. This is equivalent to killing the Bessel process with linear drift at the rate $r(x)=\frac{\sigma^{2}}{4} x^{2}$. From Eq. (56) we see that adding the killing only changes the coefficient in front of the $y^{2}$ term in the corresponding Schrödinger equation, and the spectral representation for the CIR term structure model follows immediately. A detailed spectral analysis of the CIR model can be found in Davydov and Linetsky [19, Section 4] and Gorovoi and Linetsky [34, Section 5].

\subsubsection{The 3/2 model}

Let $\left\{X_{t}, t \geq 0\right\}$ be a Bessel process with $\nu>1$ and with linear drift with $c<0$ and $\alpha=\beta=0$. For $\sigma>0$, the reciprocal squared process $\left\{\frac{4}{\sigma^{2}} X_{t}^{-2}, t \geq 0\right\}$ is a diffusion on $(0, \infty)$ with infinitesimal parameters

$$
\begin{aligned}
a(x) & =\sigma x^{3 / 2}, \quad b(x)=\kappa(\theta-x) x, \\
\kappa & :=\frac{\sigma^{2}}{2}(\nu-1)>0, \quad \theta:=-\frac{4 c}{\sigma^{2}(\nu-1)}>0 .
\end{aligned}
$$

This diffusion with non-linear drift and infinitesimal variance $\sigma^{2} x^{3}$ is the reciprocal of the square-root CIR process. This process was proposed by Cox, Ingersoll and Ross [14, p. 402, Eq. (50)] as a model for the inflation rate in their three-factor inflation model. They were able to solve the three-factor valuation PDE for the real value of a nominal bond [Eqs. (53-54)]. More recently this diffusion appeared in Lewis [56], Heston [40], Ahn and Gao [2] and Lewis [58] in different contexts. Heston [40] and Lewis [58] apply this process in the context of stochastic volatility models. The latter reference provides a detailed study of a stochastic volatility model where the instantaneous asset price variance follows this process. Lewis [56] and Ahn and Gao [2] propose this process as a model for the nominal short rate. They show that the $3 / 2$ model is more empirically plausible than the square-root model. The accumulated empirical evidence estimating short rate models with the diffusion parameter $\sim r^{\gamma}$ suggests that empirically $\gamma>1$, thus contradicting the square-root specification. Furthermore, recent empirical studies suggest that the short rate drift is substantially non-linear in the short rate. Lewis [56] (see also [2]) obtains an analytical solution for the zero-coupon bond price by directly solving the PDE. This solution is, in fact, contained in a more general solution given in Eq. (54) of Cox, Ingersoll and Ross [14] for their three-factor inflation model. As an illustration of the spectral method, below we work out explicit expressions for 
eigenvalues and eigenfunctions in the $3 / 2$ term structure model that immediately lead to solutions for the state-price density, zero-coupon bonds and bond options.

Suppose the short rate $\left\{r_{t}, t \geq 0\right\}$ follows a diffusion with infinitesimal parameters (58) with the initial short rate $r_{0}=r>0$ and $\sigma, \kappa, \theta>0$. The spectrum is purely discrete. The SL equation is:

$$
\frac{1}{2} \sigma^{2} r^{3} u^{\prime \prime}+\kappa(\theta-r) r u^{\prime}+(\lambda-r) u=0 .
$$

Introduce the following notation:

$$
\alpha:=\frac{\kappa}{\sigma^{2}}+1, \quad \beta:=\frac{2 \kappa \theta}{\sigma^{2}}, \quad m:=\sqrt{\left(\frac{\kappa}{\sigma^{2}}+\frac{1}{2}\right)^{2}+\frac{2}{\sigma^{2}}} .
$$

The speed density is: $\mathfrak{m}(r)=\frac{2}{\sigma^{2}} r^{-2 \alpha-1} e^{-\frac{\beta}{r}}$. The substitution

$$
u(r)=r^{\alpha} e^{\frac{\beta}{2 r}} w\left(\frac{\beta}{r}\right)
$$

reduces Eq. (59) to Whittaker's form of the confluent hypergeometric equation $(\xi:=\beta / r)$ :

$$
w^{\prime \prime}+\left(-\frac{1}{4}+\frac{\frac{\lambda}{\kappa \theta}+\alpha}{\xi}+\frac{\frac{1}{4}-m^{2}}{\xi^{2}}\right) w=0 .
$$

The solutions of Lemma 1 are expressed in terms of the Whittaker functions $[9,83]$ :

$$
\phi(r, \lambda)=r^{\alpha} e^{\frac{\beta}{2 r}} M_{\frac{\lambda}{\kappa \theta}+\alpha, m}\left(\frac{\beta}{r}\right), \quad \psi(r, \lambda)=r^{\alpha} e^{\frac{\beta}{2 r}} W_{\frac{\lambda}{\kappa \theta}+\alpha, m}\left(\frac{\beta}{r}\right) .
$$

Their Wronskian is:

$$
w(\lambda)=-\frac{\beta \Gamma(2 m+1)}{\Gamma\left(m-\frac{\lambda}{\kappa \theta}-\alpha+\frac{1}{2}\right)} .
$$

The eigenvalues are found as zeros of the Wronskian (here it is more convenient for us to label the eigenvalues starting from zero; the zeros of the Wronskian are the zeros of the reciprocal of the Gamma function $\left.1 / \Gamma\left(m-\frac{\lambda}{\kappa \theta}-\alpha+\frac{1}{2}\right)\right)$ :

$$
\lambda_{n}=\kappa \theta(n+m-\alpha+1 / 2), \quad n=0,1,2, \ldots .
$$

Substituting $\lambda_{n}$ in Eqs. (61), the Whittaker functions $M_{m+n+1 / 2, m}(z)$ and $W_{m+n+1 / 2, m}(z)$ reduce to the generalized Laguerre polynomials $[9$, p. 214] and the functions

$$
\begin{gathered}
\phi\left(r, \lambda_{n}\right)=\frac{n ! \Gamma(2 m+1) \beta^{m+1 / 2}}{\Gamma(2 m+n+1)} r^{\alpha-m-1 / 2} L_{n}^{(2 m)}\left(\frac{\beta}{r}\right) \\
\psi\left(r, \lambda_{n}\right)=(-1)^{n} n ! \beta^{m+1 / 2} n ! r^{\alpha-m-1 / 2} L_{n}^{(2 m)}\left(\frac{\beta}{r}\right)
\end{gathered}
$$

become linearly dependent, $\phi\left(r, \lambda_{n}\right)=A_{n} \psi\left(r, \lambda_{n}\right)$ with

$$
A_{n}=(-1)^{n} \frac{\Gamma(2 m+1)}{\Gamma(2 m+n+1)} .
$$


The Wronskian derivative $w^{\prime}\left(\lambda_{n}\right)$ at an eigenvalue is

$$
w^{\prime}\left(\lambda_{n}\right)=(-1)^{n} \frac{2}{\sigma^{2}} n ! \Gamma(2 m+1) .
$$

The normalized eigenfunctions (34) are:

$$
\varphi_{n}(r)=\mathcal{N}_{n} r^{\alpha-m-1 / 2} L_{n}^{(2 m)}\left(\frac{\beta}{r}\right), \quad \mathcal{N}_{n}^{2}=\frac{\sigma^{2} \beta^{2 m+1} n !}{2 \Gamma(2 m+n+1)}, \quad n=0,1,2, \ldots .
$$

The state-price density of the $3 / 2$ term structure model has the spectral representation:

$$
\begin{aligned}
p\left(t ; r, r_{t}\right)= & \beta^{2 m+1} r^{\alpha-m-1 / 2} r_{t}^{-\alpha-m-3 / 2} e^{-\frac{\beta}{r_{t}}} \\
& \times \sum_{n=0}^{\infty} e^{-\kappa \theta[n+m-\alpha+1 / 2] t} \frac{n !}{\Gamma(2 m+n+1)} L_{n}^{(2 m)}\left(\frac{\beta}{r}\right) L_{n}^{(2 m)}\left(\frac{\beta}{r_{t}}\right) .
\end{aligned}
$$

Applying the Hille-Hardy summation formula for the generalized Laguerre polynomials [24, p. 189, Eq. (20)], the state-price density takes the form:

$$
\begin{aligned}
p\left(t ; r, r_{t}\right)= & \frac{\beta}{\left(1-e^{-\kappa \theta t}\right) r_{t}^{2}}\left(\frac{e^{\kappa \theta t} r}{r_{t}}\right)^{\alpha-\frac{1}{2}} \\
& \times \exp \left\{-\frac{\beta\left(e^{-\kappa \theta t} r^{-1}+r_{t}^{-1}\right)}{1-e^{-\kappa \theta t}}\right\} I_{2 m}\left(\frac{\beta}{\sqrt{r r_{t}} \sinh \left(\frac{\kappa \theta t}{2}\right)}\right),
\end{aligned}
$$

where $I_{\nu}(z)$ is the modified Bessel function.

Let $P(t, r)$ be the time-zero price of a zero-coupon bond with maturity $t$. The Fourier coefficients for the spectral expansion for the bond price,

$$
P(t, r)=\sum_{n=0}^{\infty} c_{n} e^{-\lambda_{n} t} \varphi_{n}(r)
$$

are obtained in closed form by computing the inner product $c_{n}=\left(1, \varphi_{n}\right)$ with the help of Gradshteyn and Ryzhik [35, p. 850, Eq. (11)]:

$$
c_{n}=\frac{2}{\sigma^{2}} \mathcal{N}_{n} \beta^{-\alpha-m-\frac{1}{2}} \frac{\Gamma(\alpha+m+1 / 2) \Gamma(m+n-\alpha+1 / 2)}{n ! \Gamma(m-\alpha+1 / 2)} .
$$

Substituting the coefficients in the expansion (63) and using the summation formula for Laguerre polynomials [80, p. 704, Eq. (16)], we obtain a closed-form expression for the zero-coupon bond price in the $3 / 2$ model:

$$
\begin{gathered}
P(r, t)=\frac{\Gamma(b-a)}{\Gamma(b)}\left(\frac{z(t)}{r}\right)^{a} M\left(a, b,-\frac{z(t)}{r}\right) \\
a:=m-\alpha+1 / 2, \quad b:=2 m+1, \quad z(t):=\frac{2 \kappa \theta}{\sigma^{2}\left(e^{\kappa \theta t}-1\right)}
\end{gathered}
$$


and $M(a, b, x)$ is the Kummer confluent hypergeometric function [83]. This expression agrees with Eq. (54) in Cox, Ingersoll and Ross [14] and Eq. (9) in Lewis [56]. The same result is obtained by integrating the unit payoff against the state-price density (62) and using Gradstein and Ryzhik [35, p. 741, Eq. (2)].

Having an analytical expression for the state-price density, any interest rate derivative can be priced by integrating the payoff against the state-price density:

$$
\mathbb{E}_{r}\left[e^{-\int_{0}^{t} r_{u} d u} f\left(r_{t}\right)\right]=\int_{0}^{\infty} f\left(r_{t}\right) p\left(t ; r, r_{t}\right) d r_{t} .
$$

For example, for a put option expiring at time $t$ on the zero-coupon bond with maturity $T>t$, the payoff can be written in the form $f\left(r_{t}\right)=\left(K-P\left(r_{t}, T-\right.\right.$ t)) $\mathbf{1}_{\left\{r_{t}>x^{*}\right\}}$, where $x^{*}$ is the unique solution of the equation $P\left(x^{*}, T-t\right)=K$ (to be found numerically) and $P(t, r)$ is the zero-coupon bond value function (64). The one-dimensional integral in $r_{t}$ is computed numerically.

\subsubsection{The CEV model}

Let $\left\{X_{t}, t \geq 0\right\}$ be a Bessel process with $\nu<0$ and with linear drift with $c \in \mathbb{R}$. If $\nu>-1$ the origin is regular and we send the process to the cemetery state $\partial$ at the first hitting time of zero, $\mathcal{T}_{0}$. If $\nu \leq-1$, the origin is exit. Thus, in both cases the lifetime of the process is $\zeta=\mathcal{T}_{0}$. For some $\delta>0$ define a new process $\left\{S_{t}, t \geq 0\right\}$ as follows:

$$
S_{t}:=\left\{\begin{array}{cl}
\left(\frac{\delta}{2|\nu|} X_{t}\right)^{-2 \nu}, & 0 \leq t<\zeta \\
\partial, & t \geq \zeta
\end{array} .\right.
$$

This process is a diffusion on $(0, \infty)$ with infinitesimal parameters

$a(x)=\sigma(x) x=\delta x^{1+\beta}, \quad b(x)=\mu x$, where $\beta:=\frac{1}{2 \nu}<0, \quad \mu:=-2 c \nu \in \mathbb{R}$.

This is a constant elasticity of variance (CEV) model of Cox [13] (see also [17] and references therein). The CEV specification nests the Samuelson-Black-ScholesMerton geometric Brownian motion model $(\beta=0)$ and the absolute diffusion $(\beta=$ $-1)$ and square-root $(\beta=-1 / 2)$ models of Cox and Ross [15]. For $\beta<0$, the local volatility $\sigma(x)=\delta x^{\beta}$ is a decreasing function of the asset price. We have two model parameters: $\beta$ is the elasticity of the local volatility function, $\beta=x \sigma^{\prime} / \sigma$, and $\delta$ is the scale parameter. Cox originally restricted $\beta$ to the range $-1 \leq \beta \leq 0$. However, Jackwerth and Rubinstein [43] empirically find that typical values of $\beta$ implicit in the post-crash of $1987 \mathrm{~S} \& \mathrm{P} 500$ stock index option prices are as low as $\beta=-4$. They call the model with $\beta<-1$ unrestricted $C E V$. For $\beta<0,+\infty$ is a natural boundary; attracting (non-attracting) for $\mu>0(\mu \leq 0)$. For $-1 / 2 \leq \beta<0$ $(-\infty<\beta<-1 / 2)$, the origin is exit (regular killing). The process with $\beta<0$ is used to model the volatility smile/skew phenomenon in equity options markets. Davydov and Linetsky [19] and Linetsky [62] provide detailed spectral analysis of 
the CEV process and obtain analytical solutions for vanilla, barrier, and lookback options (see [7] for the trinomial lattice valuation in the CEV model).

\subsection{Coulomb potential, Bessel processes with constant drift and two non-linear term structure models}

\subsubsection{Coulomb potential and Bessel processes with constant drift}

Consider a diffusion with the infinitesimal parameters

$$
\begin{aligned}
& a(x)=1, \quad b(x)=\frac{\nu+\frac{1}{2}}{x}+\mu, \quad r(x)=\frac{\alpha^{2}}{2 x^{2}}+\frac{\beta}{x}, \\
& x \in(0, \infty), \quad \nu, \mu \in \mathbb{R}, \quad \alpha, \beta \geq 0 .
\end{aligned}
$$

The Liouville transformation (25) with $x_{0}=0$ reduces the SL Eq. (23) to the Schrödinger equation with Coulomb potential [73, p. 1663]:

$$
Q(y)=\frac{\nu^{2}+\alpha^{2}-\frac{1}{4}}{y^{2}}+\frac{\sqrt{2}[(\nu+1 / 2) \mu+\beta]}{y}+\frac{\mu^{2}}{2}, \quad y \in(0, \infty) .
$$

First consider the case with $\alpha=\beta=0$. For $\nu=d / 2-1$ with some integer $d \geq 2$ this process arises as a radial part of the $d$-dimensional process with the infinitesimal generator

$$
\frac{1}{2} \Delta+\mu \frac{x}{|x|} \cdot \nabla
$$

For $d=2$ and $\mu<0$ this process was studied by Kendall [47], where it was called $a$ pole seeking Brownian motion (see also [77, pp. 362-364] and [89, p. 104]). Here we consider this process with $\nu, \mu \in \mathbb{R}$ and call it a Bessel process with constant drift (in contrast with the Bessel process with linear drift $c x$ in Sec. 6.3). The boundary classification at 0 is independent of $\mu$ and is the same as for the standard Bessel process. For all $\nu \in \mathbb{R},+\infty$ is O-NO natural with cutoff $\Lambda=\mu^{2} / 2$. According to Theorem $3,+\infty$ is non-oscillatory (oscillatory) for $\lambda=\Lambda=\mu^{2} / 2$ if $\mu(\nu+1 / 2) \geq 0$ $(\mu(\nu+1 / 2)<0)$. Thus, we are in the Spectral Category II with purely absolutely continuous spectrum above $\mu^{2} / 2$. If $\mu(\nu+1 / 2)<0$ we have an infinite sequence of eigenvalues clustering at $\mu^{2} / 2$ (i.e., $\mu^{2} / 2$ is the limit point of the point spectrum). If $\mu(\nu+1 / 2) \geq 0$, there may only be a finite set of non-negative eigenvalues in $\left[0, \mu^{2} / 2\right]$. A practically important property is that the SL equation for the process killed at the rate $r(x)=\frac{\alpha^{2}}{2 x^{2}}+\frac{\beta}{x}$ reduces to the Schrödinger equation with potential function of the same functional form - only the coefficients in front of the $y^{-1}$ and $y^{-2}$ change. The Schrödinger equation with Coulomb potential (68) has the form of the Whittaker Eq. (60) with the Whittaker functions as solutions.

Remark. Yor [89] calls the process with the infinitesimal generator

$$
\mathcal{G}=\frac{1}{2} \frac{d^{2}}{d x^{2}}+\left(\frac{\nu+\frac{1}{2}}{x}-\delta\right) \frac{d}{d x} .
$$


Bessel process with "naive" $\operatorname{drift} \delta>0$ in order to avoid confusion with the diffusion obtained by taking the radial part of an $\mathbb{R}^{d}$-valued Brownian motion started at the origin and with some drift vector $\vec{\delta}$ and with the infinitesimal generator

$$
\frac{1}{2} \Delta+\vec{\delta} \cdot \nabla
$$

This latter diffusion has the infinitesimal generator

$$
\mathcal{G}=\frac{1}{2} \frac{d^{2}}{d x^{2}}+\left(\frac{\nu+\frac{1}{2}}{x}+\frac{\delta I_{\nu+1}(\delta x)}{I_{\nu}(\delta x)}\right) \frac{d}{d x},
$$

where $\nu=d / 2-1, \delta=|\vec{\delta}|$ and $I_{\nu}(z)$ is the Bessel function of order $\nu$, and is usually called Bessel process with drift $[77,86]$. Here we call the former process Bessel process with constant drift to emphasize that the additional drift term is a constant, and also to avoid confusion with the diffusion of Sec. 6.3 we refer to as Bessel process with linear drift. ${ }^{\mathrm{b}}$

\subsubsection{Two non-linear term structure models}

Let $\left\{X_{t}, t \geq 0\right\}$ be a Bessel process with $\nu>1$ and constant drift $\mu<0$ and $\alpha=\beta=0$. For $\sigma>0$, the reciprocal squared process $\left\{\frac{4}{\sigma^{2}} X_{t}^{-2}, t \geq 0\right\}$ is a diffusion on $(0, \infty)$ with the infinitesimal parameters

$$
\begin{aligned}
a(x) & =\sigma x^{3 / 2}, \quad b(x)=\kappa\left(\theta-x^{\frac{1}{2}}\right) x^{\frac{3}{2}}, \\
\kappa & :=\frac{\sigma^{2}}{2}(\nu-1)>0, \quad \theta:=-\frac{2 \mu}{\sigma(\nu-1)}>0 .
\end{aligned}
$$

This diffusion with infinitesimal variance $\sigma^{2} x^{3}$ is similar to the $3 / 2$ model of Sec. 6.3 .3 but has a different specification of non-linear drift. The spectral representation of the state-price density in the term structure model with the short rate following this diffusion is obtained by killing the process at the linear rate $r(x)=x$. This corresponds to killing the Bessel process with constant drift at the rate $r(x)=\frac{4}{\sigma^{2}} x^{-2}$. Transforming to the Liouville normal form, only the coefficient in front of the $y^{-2}$ is modified. Thus, in this term structure model the spectral representation for the state price density follows immediately from the spectral representation for the transition density for the Bessel process with constant negative drift.

Let $\left\{X_{t}, t \geq 0\right\}$ be a Bessel process with $\nu \geq 1 / 2$ and constant drift $\mu<0$ and $\alpha=\beta=0$. For some $\sigma>0$, the reciprocal process $\left\{\left(\sigma X_{t}\right)^{-1}, t \geq 0\right\}$ is a diffusion on $(0, \infty)$ with the infinitesimal parameters

$$
\begin{aligned}
a(x) & =\sigma x^{2}, \quad b(x)=\kappa(\theta-x) x^{2}, \\
\kappa & :=\sigma^{2}(\nu-1 / 2)>0, \quad \theta:=-\frac{\mu}{\sigma(\nu-1 / 2)}>0 .
\end{aligned}
$$

\footnotetext{
${ }^{\mathrm{b}}$ The author thanks Marc Yor for bringing the reference [89] to his attention.
} 
This diffusion has the CEV infinitesimal variance $\sigma^{2} x^{2 \gamma}$ with $\gamma=2$ in contrast to the $3 / 2$ model. Its drift is also more non-linear that the $3 / 2$ model. In light of the recent empirical evidence on non-linearity of the drift and high positive values for $\gamma$, this model may be expected to outperform the $3 / 2$ model in empirical tests. The spectral representation of the state-price density is obtained by killing the process at the rate $r(x)=x$. This corresponds to killing the Bessel process with constant drift at the rate $r(x)=(\sigma x)^{-1}$. Transforming to the Liouville normal form, only the coefficient in front of $y^{-1}$ is modified. Thus, in this term structure model the spectral representation for the state price density follows immediately from the spectral representation for the transition density for the Bessel process with constant negative drift. More details on Bessel processes with constant drift and the associated non-affine term structure models can be found in Linetsky [63].

\subsection{Morse potential, geometric Brownian motion with affine drift, arithmetic Asian options, Merton's cash dividends, GARCH stochastic volatility, energy options and interest rate models}

\subsubsection{Morse potential and geometric Brownian motion with affine drift}

Consider a diffusion with the infinitesimal parameters

$$
a(x)=\sigma x, \quad b(x)=A x+B, \quad x \in(0, \infty), \quad \sigma>0, \quad A, B \in \mathbb{R}, \quad B \neq 0 .
$$

Assume initially $r(x)=0$. For $B=0$ this process reduces to the geometric Brownian motion discussed in Sec. 6.1. For $B \neq 0$ we call this process geometric Brownian motion with affine drift. This process was studied by Wong [88] who obtained a spectral representation for $B>0$ and $A<\sigma^{2} / 2$. For all $A, B \in \mathbb{R},+\infty$ is a natural boundary. Adding the constant $B \neq 0$ in the drift drastically changes behavior of the process near the origin. For all $A \in \mathbb{R}, 0$ is exit (entrance) for $B<0(B>0)$. For $B>0$ and $A<\frac{\sigma^{2}}{2}$ (the case studied by [88]), the process has a stationary distribution with the reciprocal Gamma density:

$$
\pi(x) \sim x^{\frac{2 A}{\sigma^{2}}-2} e^{-\frac{2 B}{\sigma^{2} x}} .
$$

Let $\zeta:=\mathcal{T}_{0}$ be the lifetime of the process $(\zeta=\infty$ if $B>0)$. The process $\left\{\ln X_{t}, 0 \leq\right.$ $t<\zeta\}$ is a diffusion with the infinitesimal parameters

$$
a(x)=\sigma, \quad b(x)=A-\frac{1}{2} \sigma^{2}+B e^{-x} .
$$

The Liouville transformation (25) with $x_{0}=0$ reduces the corresponding SL equation to the Schrödinger equation with Morse potential [72, 73]:

$$
\begin{gathered}
Q(y)=c_{0}+c_{1} e^{-\gamma y}+c_{2} e^{-2 \gamma y}, y \in(0, \infty) \\
c_{0}=\frac{1}{2 \sigma^{2}}\left(A-\frac{1}{2} \sigma^{2}\right)^{2}, \quad c_{1}=B\left(\frac{A}{\sigma^{2}}-1\right), \quad c_{2}=\frac{B^{2}}{2 \sigma^{2}}, \gamma:=\sqrt{2} \sigma .
\end{gathered}
$$


The Schrödinger equation with potential of the form $a e^{-2 c x}+b e^{-c x}$ first appeared in Morse [72]. The origin is NONOSC and $+\infty$ is O-NO with cutoff $\Lambda=c_{0}$. The cutoff value is non-oscillatory. Thus we have an absolutely continuous spectrum above $c_{0}$ plus a finite set of non-negative eigenvalues below $c_{0}$ (it is empty for some parameter values).

\subsubsection{Arithmetic Asian options}

Arithmetic Asian (average price) options deliver payoffs based on the average price of the underlying asset between the contract inception and expiration. Suppose the underlying asset price follows geometric Brownian motion process $S_{t}=S_{0} e^{\left(r-q-\sigma^{2} / 2\right) t+\sigma B_{t}}$, where $\left\{B_{t}, t \geq 0\right\}$ is a standard Brownian motion. The Asian-style put option has the payoff at expiration $T>0$ : $\left(K-\frac{1}{T} \int_{0}^{T} S_{t} d t\right)^{+}$. Thus, to price the Asian put option we need to compute the expectation

$$
\mathbb{E}\left[\left(K-\frac{1}{T} \int_{0}^{T} S_{t} d t\right)^{+}\right]
$$

Set $\nu:=\frac{2}{\sigma^{2}}\left(r-q-\frac{\sigma^{2}}{2}\right)$ and for $t>0$ define

$$
A_{t}^{(\nu)}:=\int_{0}^{t} e^{2\left(B_{u}+\nu u\right)} d u
$$

By scaling, the problem is reduced to the calculation of the expectation [29]:

$$
\mathbb{E}\left[\left(k-A_{\tau}^{(\nu)}\right)^{+}\right], \quad \tau:=\frac{\sigma^{2} T}{4}, \quad k:=\frac{\tau K}{S_{0}} .
$$

For fixed $t>0$, one can show that the random variable $A_{t}^{(\nu)}$ has the same distribution as the state $X_{t}$ of a diffusion process with the infinitesimal parameters

$$
a(x)=2 x, \quad b(x)=2(\nu+1) x+1
$$

and started at the origin at time zero, $X_{0}=0$ (see [21] and references therein). Thus, the problem of pricing continuously-sampled arithmetic Asian options is reduced to the problem of pricing options on the one-dimensional diffusion (73). An alternative derivation based on the similarity reduction of the Asian option pricing PDE with two state variables (spot price and average) to the PDE with one state variable was given by Lipton [65]. The call payoff is not square-integrable with the speed density of the diffusion (73), while the put payoff is. The spectral expansion for the arithmetic Asian put is computed in Linetsky $[60,61]$ in terms of the spectral expansion (see [12]) for closely related results in a different setting). The arithmetic Asian call is then priced by appealing to the call-put parity relationship. 


\subsubsection{Merton's cash dividends}

Let $\left\{S_{t}, t \geq 0\right\}$ be the risk-neutral price process for an asset with constant volatility $\sigma>0$ that continuously pays cash dividends at the rate of $D>0$ dollars per year. It is a diffusion with infinitesimal parameters $(r>0$ is the constant risk-free rate):

$$
a(x)=\sigma x, \quad b(x)=r x-D .
$$

Similar to the arithmetic Asian option application, it is a diffusion of the general form (71). The difference is that here $B=-D<0$ and, hence, zero is exit (the asset price hits zero in finite time and is sent to the bankruptcy state with probability one), while in the Asian option application $B=1>0$ and zero is entrance. The problem of pricing options on assets that continuously pay cash dividends has been first studied by Merton [70] who obtained an asymptotic result for the call option price with infinite time to maturity. Lewis [57] has recently obtained closed-form solutions for call and put options with finite time to maturity in terms of spectral expansions. As mentioned in the remark in Sec. 3, in this case neither call nor put payoffs are square-interable with the speed density, and the analysis requires special care. The close connection between the Asian option problem and the cash dividends problem was also emphasized by Lipton [65].

\subsubsection{GARCH stochastic volatility model, spot energy model, and Brennan and Schwartz [8] interest rate model}

Under a GARCH diffusion specification $[58,74]$ the infinitesimal variance $V_{t}=\sigma_{t}^{2}$ of the asset return is assumed to follow a positive mean-reverting diffusion process solving the SDE

$$
d V_{t}=\kappa\left(\theta-V_{t}\right) d t+\xi V_{t} d B_{t}, \quad V_{0}=\sigma_{0}^{2},
$$

where $\theta>0$ is the long-run variance level, $\xi>0$ is the volatility of variance, and $\kappa>0$ is the rate of mean-reversion. This process belongs to the family (71). It possesses a stationary distribution with the reciprocal Gamma density. Re-scaling and time-changing, the process $\left\{X_{t}:=\alpha V_{\frac{4 t}{\xi^{2}}}, t \geq 0\right\}$, where $\alpha=\frac{\xi^{2}}{4 \kappa \theta}$, is a diffusion with the infinitesimal parameters (73) starting at $X_{0}=\alpha V_{0}>0$ with

$$
\nu=-1-\frac{4 \kappa}{\xi^{2}}<-1 \text {. }
$$

Hence, the spectral representation for the transition density of the GARCH stochastic volatility process is immediately obtained from the spectral expansion for the process with the infinitesimal parameters (73) started at a positive value (recall that in the Asian option application the diffusion (73) is started at the origin) which, in turn, follows from the spectral expansion for the Schrödinger operator with Morse potential. The result for the GARCH diffusion transition density reads:

$$
p(t ; x, y)=\alpha p^{(\nu)}\left(\xi^{2} t / 4 ; \alpha x, \alpha y\right),
$$


where $p^{(\nu)}(t ; x, y)$ is the transition density of the diffusion (73) started at $x>0$ $[12,60]$ :

$$
\begin{aligned}
p^{(\nu)}(t ; x, y)= & \frac{1}{2 \pi^{2}} \int_{0}^{\infty} e^{-\frac{\left(\nu^{2}+\rho^{2}\right) t}{2}} e^{\frac{1}{4 x}-\frac{1}{4 y}}\left(\frac{y}{x}\right)^{\frac{\nu-1}{2}} W_{-\frac{\nu}{2}+\frac{1}{2}, \frac{i \rho}{2}}\left(\frac{1}{2 x}\right) W_{-\frac{\nu}{2}+\frac{1}{2}, \frac{i \rho}{2}}\left(\frac{1}{2 y}\right) \\
& \times\left|\Gamma\left(\frac{\nu+i \rho}{2}\right)\right|^{2} \sinh (\pi \rho) \rho d \rho+\sum_{n=0}^{\left[\frac{-\nu}{2}\right]} e^{-2 n(-\nu-n) t} \frac{2(-\nu-2 n) n !}{\Gamma(1-\nu-n)} \\
& \times e^{-\frac{1}{2 y}}\left(\frac{1}{2 x}\right)^{-n}\left(\frac{1}{2 y}\right)^{1-\nu-n} L_{n}^{(-\nu-2 n)}\left(\frac{1}{2 x}\right) \\
& \times L_{n}^{(-\nu-2 n)}\left(\frac{1}{2 y}\right), \quad x, y>0,
\end{aligned}
$$

where $W_{k, m}(z)$ is the Whittaker function, $L_{n}^{(\alpha)}(z)$ are the generalized Laguerre polynomials, and $[x]$ denotes the integer part of $x$. The summation is over a finite number of discrete eigenvalues and the integral is with respect to the continuous spectrum.

In a separate application, in the energy markets the spot price is often modeled as a mean-reverting positive diffusion (e.g., [76, p. 64])

$$
d S_{t}=\kappa\left(L-S_{t}\right) d t+\sigma S_{t} d B_{t}, \quad S_{0}=x>0,
$$

where $L>0$ is the long-run equilibrium spot price level, $\sigma>0$ is the spot price volatility and $\kappa>0$ is the rate of mean-reversion. This is the same process as in the GARCH diffusion model, and the GARCH transition density yields the spectral expansion for the energy option on integration with the option payoff.

The same diffusion is also known in the interest rate literature as the BrennanSchwartz [8] short rate model. While the spectral representation for the transition density for this model is available in closed form, there is no analytical solution for the state-price density and, hence, zero-coupon bonds. If we kill this process at the linear rate $r(x)=x$, the resulting SL equation leads to the Schrödinger equation with a three-term potential of the form $c_{1} e^{-\gamma y}+c_{2} e^{-2 \gamma y}+c_{3} e^{\gamma y}$. To the best of our knowledge, there is no closed-form solution in terms of classical special functions.

\subsubsection{Two non-linear term structure models}

For $A<\frac{\sigma^{2}}{2}$ and $B>0$ let $\left\{X_{t}, t \geq 0\right\}$ be a diffusion with the infinitesimal parameters $(71)$ and consider a reciprocal process $\left\{\left(\sigma X_{t}\right)^{-1}, t \geq 0\right\}$. It is a diffusion on $(0, \infty)$ with the infinitesimal parameters:

$$
a(x)=\sigma x, \quad b(x)=\kappa(\theta-x) x, \quad \kappa:=\sigma B>0, \quad \theta:=\frac{\sigma^{2}-A}{\sigma B}>0 .
$$

This process has been deduced by Merton [71] as a model for the short rate in his economic growth model. Lewis [57] has recently obtained closed-form solutions for zero-coupon bonds in this model in terms of spectral expansions. 
Consider a squared reciprocal process $\left\{\left(\sigma X_{t}\right)^{-2}, t \geq 0\right\}$. It is a diffusion on $(0, \infty)$ with infinitesimal parameters:

$$
a(x)=2 \sigma x, \quad b(x)=\kappa\left(\theta-x^{\frac{1}{2}}\right) x, \quad \kappa:=2 \sigma B>0, \quad \theta:=\frac{\frac{3}{2} \sigma^{2}-A}{\sigma B}>0 .
$$

This process provides another analytically tractable specification for the short rate with non-linear drift.

\subsection{Volatility smile, hypergeometric diffusions and modified Pöschl-Teller and hyperbolic barrier potentials}

\subsubsection{Hypergeometric diffusions}

Our final example is a diffusion with the infinitesimal parameters

$$
a(x)=\sqrt{A x^{2}+B x+C}, \quad b(x)=\mu x, \quad A, C \geq 0, \quad B^{2}<4 A C, \quad \mu \in \mathbb{R} .
$$

For $B^{2}<4 A C$ the parabola $A x^{2}+B x+C$ is above the $x$-axis for all real $x$. Hence, a diffusion $\left\{X_{t}, t \geq 0\right\}$ with the infinitesimal parameters (77) can be defined on the whole real line. We call this process hypergeometric diffusion since solutions to the associated SL equation are expressed in terms of the Gauss hypergeometric function. This diffusion was first studied by Wong [88] in a special case.

The process $\left\{Z_{t}:=\frac{2 A X_{t}+B}{\sqrt{4 A C-B^{2}}}, t \geq 0\right\}$ is a diffusion on $\mathbb{R}$ with the infinitesimal parameters:

$$
a(x)=\sqrt{A\left(x^{2}+1\right)}, \quad b(x)=\mu(x-L), \quad L:=\frac{B}{\sqrt{4 A C-B^{2}}} .
$$

The process $\left\{Y_{t}:=\operatorname{arcsinh}\left(Z_{t}\right), t \geq 0\right\}$ is a diffusion on $\mathbb{R}$ with the infinitesimal parameters:

$$
a(x)=\sqrt{A}, \quad b(x)=\left(\mu-\frac{1}{2} A\right) \tanh x-\frac{\mu L}{\cosh x} .
$$

The Liouville transformation with $x_{0}=0$ reduces the associated SL equation to the Schrödinger equation with potential:

$$
\begin{gathered}
Q(y)=c_{0}+\frac{c_{1}}{\cosh ^{2}(\gamma y)}+c_{2} \frac{\sinh (\gamma y)}{\cosh ^{2}(\gamma y)} \\
\gamma:=\frac{A}{\sqrt{2}}, \quad c_{0}:=\frac{1}{2}\left(\frac{\mu}{A}-\frac{1}{2}\right)^{2}, \quad c_{1}:=\frac{\mu^{2} L^{2}}{2 A}-\frac{1}{2 A}\left(\mu-\frac{A}{2}\right)\left(\mu-\frac{3 A}{2}\right) \\
c_{2}:=\frac{\mu L}{A}(A-\mu) .
\end{gathered}
$$

This is known as a hyperbolic barrier potential [36, p. 251]. Solutions are expressed in terms of the Gauss hypergeometric function. Both $-\infty$ and $+\infty$ are O-NO with cutoff $\Lambda_{1}=\Lambda_{2}=c_{0}(Q(y) \rightarrow \infty$ as $y \rightarrow \pm \infty)$ and we have multiplicity-two purely absolutely continuous spectrum above $c_{0}$ (similar to the standard Brownian motion 
on the real line) plus a finite set of eigenvalues below $c_{0}$ (Spectral Category III). For $\mu=0$, the potential function simplifies to:

$$
Q(y)=\frac{1}{8}-\frac{3 A}{8} \frac{1}{\cosh ^{2}(\gamma y)} .
$$

This is a special case of the modified Pöschl-Teller potential [36, pp. 244-245]. Solutions are expressed in terms of Legendre functions.

\subsubsection{An analytically tractable volatility smile model}

For the purpose of modeling non-negative asset prices, we restrict the process with the infinitesimal parameters $(77)$ to the positive half-line $(0, \infty)$ and make 0 a killing boundary by sending the process to a cemetery state $\partial$ at its lifetime $\zeta=\mathcal{T}_{0} \equiv \inf \left\{t \geq 0: X_{t}=0\right\}$. The resulting process $\left\{S_{t}, t \geq 0\right\}$ is a hybrid of Black-Scholes-Merton, square root and absolute diffusion specifications. With three volatility parameters $A, B$ and $C$, this model can be calibrated to a variety of implied volatility smile shapes. To facilitate calibration, it is convenient to parameterize the diffusion coefficient as follows:

$$
a(x)=\sigma_{K} \sqrt{\frac{x^{2}+\alpha K x+\beta K^{2}}{1+\alpha+\beta}}, \quad \sigma_{K}>0, \quad K>0, \quad \alpha \in \mathbb{R}, \quad \beta>\frac{\alpha^{2}}{4},
$$

so that $a(K)=\sigma_{K} K$. Here $K$ is some reference asset price level (e.g., the asset price level at the time of calibration), $\sigma_{K}$ is the local volatility at the reference level (ATM volatility), and $\alpha$ and $\beta$ are two model parameters governing the shape of the local volatility function

$$
\sigma(x):=\frac{a(x)}{x}=\sigma_{K} \sqrt{\frac{1+\alpha(K / x)+\beta(K / x)^{2}}{1+\alpha+\beta}} .
$$

\subsection{Currency target zones, Jacobi diffusion and Pöschl-Teller-type potentials}

Larsen and Sorensen [52] has recently proposed an analytically tractable model for a foreign exchange rate in a target zone (which the central banks involved promissed to keep within fixed bounds) and applied it to the empirical analysis of the European Monetary System from 1979 until the introduction of the Euro in 1999. They model a spot exchange rate as a diffusion process $S$ that may not be more than $a \times 100$ percent over the central parity $\mu$ for any of the two currencies, i.e., $\mu /(1+a)<S_{t}<\mu(1+a)$. Then $X_{t}=\ln \left(S_{t}\right)$ must satisfy that $m-z<X_{t}<m+z$, where $m=\ln \mu$ and $z=\ln (1+a)$. The logarithm of the spot exchange rate is modeled as a diffusion

$$
d X_{t}=\beta\left[(m+\gamma z)-X_{t}\right] d t+\sigma \sqrt{z^{2}-\left(X_{t}-m\right)^{2}} d B_{t},
$$


where $\beta>0$ and $\gamma \in(-1,1)$. This process reverts to its mean $m+\gamma z$. The parameter $\gamma$ is an assymetry parameter that expresses whether one currency is stronger than the other. The process is ergodic on the inverval $(m-z, m+z)$ if and only if $\beta \geq \sigma^{2}$ and $-1+\sigma^{2} / \beta \leq \gamma \leq 1-\sigma^{2} / \beta$. The stationary distribution is a shifted and re-scaled Beta. Both boundaries at $m-z$ and $m+z$ are unattainable entrance boundaries. A standardized process $\bar{X}_{t}:=\left(X_{t}-m\right) / z$ follows a Jacobi diffusion

$$
d \bar{X}_{t}=\kappa\left(\gamma-\bar{X}_{t}\right) d t+A \sqrt{1-\bar{X}^{2}} d B_{t}, \quad \kappa:=\beta z, \quad A:=\sigma z,
$$

on the interval $(-1,1)[46,88]$. The Liouville transformation reduces the associated SL equation to the Schrödinger equation with potential of the form:

$$
Q(y)=c_{0}+\frac{c_{1}}{\cos ^{2}(\gamma y)}+c_{2} \frac{\sin (\gamma y)}{\cos ^{2}(\gamma y)}, \quad \gamma:=\frac{\sigma z}{\sqrt{2}} .
$$

This potential is of the Pöschl-Teller type [36, Section 6.5, pp. 240-241]. The spectrum is purely discrete and the eigenfunctions are expressed in terms of Jacobi polynomials.

\section{Conclusion}

This paper has focused on the spectral expansion approach to the valuation of contingent claims when the underlying state variable follows a one-dimensional diffusion. Based on the Sturm-Liouville theory applied to the infinitesimal generator of the pricing semigroup, under some technical conditions, all models have been classified into three spectral categories, with the qualitative nature of the spectrum explicitly determined for each category. In order to determine the spectral representation explicitly, one needs explicit expressions for solutions of the Sturm-Liouville equation. The Liouville transformation reduces the Sturm-Liouville equation associated with a one-dimensional diffusion with diffusion coefficient $a(x)$, drift $b(x)$ and killing rate $r(x)$ to the one-dimensional Schrödinger equation with a potential function $Q(x)$ constructed from $a(x), b(x)$ and $r(x)$. If analytical solutions are available for the Schrödinger equation, inverting the Liouville transformation yields analytical solutions for the original Sturm-Liouville equation, and the spectral representation for the diffusion process can be constructed explicitly. This gives the explicit spectral decomposition of the contingent claim value function. Table 2 summarizes some analytically tractable Schrödinger equations, associated diffusion processes and their applications in finance considered in Sec. 6.

\section{Appendix: The Spectral Theorem for Self-Adjoint Semigroups in Hilbert Space}

Let $\mathcal{H}$ be a separable real Hilbert space. If $\mathcal{A}$ is a self-adjoint operator in $\mathcal{H}$, i.e., $\mathcal{A}$ is symmetric, $(\mathcal{A} f, g)=(f, \mathcal{A} g), f, g \in D(\mathcal{A})$, and the domains of $\mathcal{A}$ and its adjoint $\mathcal{A}^{*}$ in $\mathcal{H}$ coincide, $D(\mathcal{A})=D\left(\mathcal{A}^{*}\right) \subset \mathcal{H}$, we say that $\mathcal{A}$ is non-negative (non-positive) 
if $(f, \mathcal{A} f) \geq 0((f, \mathcal{A} f) \leq 0)$ for all $f \in D(\mathcal{A})$. We define a self-adjoint contraction semigroup $\left\{P_{t}, t \geq 0\right\}$ to be a one-parameter semigroup such that $P_{t}$ is a self-adjoint contraction for all $t \geq 0[16$, p. 99]. We have a generation theorem.

Theorem 6 (Generation Theorem; Davies [16, p. 100]). $\left\{P_{t}, t \geq 0\right\}$ is a self-adjoint contraction semigroup in $\mathcal{H}$ if and only if its infinitesimal generator $\mathcal{G}$ is a self-adjoint and non-positive operator in $\mathcal{H}$.

To formulate the spectral theorem for self-adjoint semigroups we need to define projection-valued measures or spectral measures (e.g., [81, Chapter VIII]).

Table 2. Summary of analytically tractable models in Sec. 6 .

\begin{tabular}{|c|c|c|}
\hline Schrödinger potential & Associated diffusions & Finance models \\
\hline $\begin{array}{l}\text { Constant potential } \\
Q(x)=\text { const }\end{array}$ & $\begin{array}{c}\text { Arithmetic Brownian motion } \\
a(x)=\text { const, } b(x)=\text { const } \\
\text { Geometric Brownian motion } \\
a(x)=\sigma x, b(x)=(r-q) x\end{array}$ & Black-Scholes \\
\hline $\begin{array}{l}\text { Harmonic oscillator } \\
Q(x)=a x^{2}+b x+c\end{array}$ & $\begin{array}{l}\text { Ornstein-Uhlenbeck process } \\
a(x)=\sigma, b(x)=\kappa(\theta-x)\end{array}$ & Vasicek model \\
\hline $\begin{array}{l}\text { Radial harmonic oscillator } \\
\qquad Q(x)=a x^{2}+b x^{-2}+c\end{array}$ & $\begin{array}{c}\text { Bessel process with linear drift } \\
a(x)=1, b(x)=(\nu+1 / 2) x^{-1}+c x \\
\text { Related diffusions } \\
a(x)=\sigma x^{1 / 2}, b(x)=\kappa(\theta-x) \\
a(x)=\sigma x^{1+\beta}, b(x)=(r-q) x \\
a(x)=\sigma x^{3 / 2}, b(x)=\kappa(\theta-x) x\end{array}$ & $\begin{array}{l}\text { CIR model } \\
\text { CEV model } \\
3 / 2 \text { model }\end{array}$ \\
\hline $\begin{array}{c}\text { Coulomb potential } \\
Q(x)=a x^{-2}+b x^{-1}+c\end{array}$ & $\begin{array}{c}\text { Bessel process with constant drift } \\
a(x)=1, b(x)=(\nu+1 / 2) x^{-1}+c \\
\text { Related diffusions } \\
a(x)=\sigma x^{2}, b(x)=\kappa(\theta-x) x^{2} \\
a(x)=\sigma x^{3 / 2}, b(x)=\kappa\left(\theta-x^{1 / 2}\right) x^{3 / 2}\end{array}$ & Non-affine models \\
\hline$Q(x)=a e^{-2 \gamma x}+b e^{-\gamma x}+c$ & $\begin{array}{c}\text { GBM with affine drift } \\
a(x)=\sigma x, b(x)=A x+B \\
\text { Related models } \\
a(x)=2 x, b(x)=2(\nu+1) x+1 \\
a(x)=\sigma x, b(x)=r x-D \\
a(x)=\sigma x, b(x)=\kappa(\theta-x) x \\
a(x)=\xi x, b(x)=\kappa(\theta-x)\end{array}$ & $\begin{array}{l}\text { Asian options } \\
\text { Cash dividends } \\
\text { Merton's IR model } \\
\text { GARCH diffusion } \\
\text { Spot energy model } \\
\text { Brennan-Schwartz }\end{array}$ \\
\hline
\end{tabular}

Hyperbolic barrier

Modified Pöschl-Teller
Hypergeometric diffusion

$Q(y)=\frac{a}{\cosh ^{2}(\gamma y)}+\frac{b \sinh (\gamma y)}{\cosh ^{2}(\gamma y)}+c \quad a(x)=\sqrt{A x^{2}+B x+C}, b(x)=\mu x \quad$ Volatility smile

Pöschl-Teller type

$Q(y)=\frac{a}{\cos ^{2}(\gamma y)}+\frac{b \sin (\gamma y)}{\cos ^{2}(\gamma y)}+c \quad a(x)=A \sqrt{1-x^{2}}, b(x)=\kappa(\gamma-x)$ 
Definition 1 (Spectral Measure). Let $\mathcal{H}$ be a separable real Hilbert space and let $\mathcal{B}(\mathbb{R})$ be a Borel $\sigma$-algebra on $\mathbb{R}$. A family of bounded linear operators $\{\mathcal{E}(B)$ : $B \in \mathcal{B}(\mathbb{R})\}$ in $\mathcal{H}$ such that

(i) Each $\mathcal{E}(B)$ is an orthogonal projection (i.e., $\mathcal{E}^{2}(B)=\mathcal{E}(B)$ and $\mathcal{E}^{*}(B)=\mathcal{E}(B)$ ).

(ii) $\mathcal{E}(\emptyset)=0, \mathcal{E}(\mathbb{R})=I d$ (Id is the identity operator in $\mathcal{H}$ ).

(iii) If $B=\cup_{n=1}^{\infty} B_{n}$ with $B_{n} \cap B_{m}=\emptyset$ if $n \neq m$, then $\mathcal{E}(B)=\sum_{n=1}^{\infty} \mathcal{E}\left(B_{n}\right)$ (where the limit involved in the infinite series is taken in the strong operator topology).

(iv) $\mathcal{E}\left(B_{1}\right) \mathcal{E}\left(B_{2}\right)=\mathcal{E}\left(B_{1} \cap B_{2}\right)$.

is called a projection-valued measure, spectral measure or the resolution of the identity.

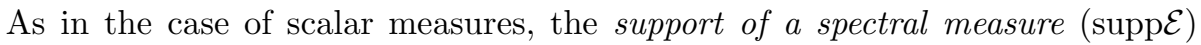
can be defined as the smallest closed subset in $\mathbb{R}$ such that $\mathcal{E}(\operatorname{supp} \mathcal{E})=I d$. For $f \in \mathcal{H}, \mu_{f}(B):=(f, \mathcal{E}(B) f)$ is a well-defined Borel measure on $\mathbb{R}$ normalized so that $\mu_{f}(\mathbb{R})=\|f\|^{2}$. Let $\mathcal{E}$ be a spectral measure and let $g$ be a real-valued Borel measurable function on $\mathbb{R}$. Then one can define the integral

$$
J_{g}:=\int_{-\infty}^{\infty} g(\lambda) \mathcal{E}(d \lambda),
$$

which is a closed operator in $\mathcal{H}$ with domain $D\left(J_{g}\right)$ dense in $\mathcal{H}$,

$$
D\left(J_{g}\right)=\left\{f \in \mathcal{H}: \int_{-\infty}^{\infty} g^{2}(\lambda) \mu_{f}(d \lambda)<\infty\right\} .
$$

The integral $J_{g}$ can be understood, for example, in the weak sense, that is, $\left(\phi, J_{g} \psi\right)=\int_{-\infty}^{\infty} g(\lambda)(\phi, \mathcal{E}(d \lambda) \psi)$ for $\phi \in \mathcal{H}$ and $\psi \in D\left(J_{g}\right)$. The operator $J_{g}$ is selfadjoint. It is bounded if and only if $g$ is bounded. The following Spectral Theorem plays a central role in the spectral analysis of self-adjoint contraction semigroups.

Theorem 7 (Spectral Theorem in the Projection-valued Measure Form; Hille and Phillips [41, Theorem 22.3.1] and Reed and Simon [81, Theorem VIII.6]). Let $\mathcal{H}$ be a separable real Hilbert space and let $\left\{P_{t}, t \geq 0\right\}$ be a strongly continuous self-adjoint contraction semigroup in $\mathcal{H}$. Then there exists a unique integral representation of $\left\{P_{t}, t \geq 0\right\}$ of the form

$$
P_{t} f=e^{t \mathcal{G}} f=\int_{(-\infty, 0]} e^{\lambda t} \mathcal{E}(d \lambda) f, \quad f \in \mathcal{H}, t \geq 0,
$$

where $\mathcal{E}$ is the spectral measure of the infinitesimal generator $\mathcal{G}$ of $P$ with $\operatorname{supp} \mathcal{E} \subset$ $(-\infty, 0]$, namely,

$$
\begin{gathered}
\mathcal{G} f=\int_{(-\infty, 0]} \lambda \mathcal{E}(d \lambda) f, \quad f \in D(\mathcal{G}), \\
D(\mathcal{G})=\left\{f \in \mathcal{H}: \int_{(-\infty, 0]} \lambda^{2} \mu_{f}(d \lambda)<\infty\right\} .
\end{gathered}
$$


For the reader's convenience we provide a collection of standard definitions and results on the structure of the spectrum of self-adjoint operators needed in the paper. We follow Reed and Simon [81], Chapters VII and VIII. In what follows $\mathcal{G}$ is a self-adjoint operator in a real separable Hilbert space $\mathcal{H}$ and $E$ is the spectral measure of $\mathcal{G}$.

- The resolvent set $\rho(\mathcal{G})$ consists of all $\lambda \in \mathbb{C}$ such that there exists a bounded operator $R_{\lambda}:=(\lambda I-\mathcal{G})^{-1}$ called the resolvent. From Theorem 6 we have the spectral representation for the resolvent of a self-adjoint contraction semigroup:

$$
R_{\alpha} f=(\alpha I-\mathcal{G})^{-1} f=\int_{(-\infty, 0]} \frac{\mathcal{E}(d \lambda) f}{\alpha-\lambda}, \quad \alpha \in \rho(\mathcal{G}), \quad f \in \mathcal{H} .
$$

- The complement $\sigma(\mathcal{G}):=\mathbb{C} \backslash \rho(\mathcal{G})$ of the resolvent set is called the spectrum of $\mathcal{G}$.

- The set $\rho(\mathcal{G})$ is open and $\sigma(\mathcal{G})$ is closed.

- If $\mathcal{G}$ is self-adjoint, then the spectrum of $\mathcal{G}$ is non-empty and lies on the real axis.

- The support of the spectral measure $\mathcal{E}$ of $\mathcal{G}$ coincides with the spectrum $\sigma(\mathcal{G})$.

- If $\mathcal{G}$ is non-positive (non-negative), then $\sigma(\mathcal{G})$ lies on the non-positive (nonnegative) half-axis.

- The pure point spectrum is defined as the set of all eigenvalues $\sigma_{p p}(\mathcal{G}):=\{\lambda \in$ $\mathbb{R}: \exists f \in D(\mathcal{G})$ such that $\mathcal{G} f=\lambda f\}$.

- One can distinguish the following subspaces in $\mathcal{H}: \mathcal{H}_{p p}$ - the closure of the linear hull of all eigenspaces of $\mathcal{G}, \mathcal{H}_{a c}$ - the set of all $f \in \mathcal{H}$ such that the measure $\mu_{f}(B)=(f, \mathcal{E}(B) f)$ is absolutely continuous with respect to the Lebesgue measure, and $\mathcal{H}_{c s}$ - the orthogonal complement of $\mathcal{H}_{p p} \oplus \mathcal{H}_{a c}$ in $\mathcal{H}$. If $f \in \mathcal{H}_{c s}$, then $\mu_{f}(B):=(f, \mathcal{E}(B) f)$ is a continuous measure singular with respect to the Lebesgue measure. The subspaces $\mathcal{H}_{p p}, \mathcal{H}_{a c}$, and $\mathcal{H}_{c s}$ are orthogonal to each other and invariant with respect to $\mathcal{G}$. The restrictions $\mathcal{G}_{p p}, \mathcal{G}_{a c}$, and $\mathcal{G}_{c s}$ of $\mathcal{G}$ to $D(\mathcal{G}) \cap \mathcal{H}_{p p}, D(\mathcal{G}) \cap \mathcal{H}_{a c}$, and $D(\mathcal{G}) \cap \mathcal{H}_{c s}$ are self-adjoint as operators in $\mathcal{H}_{p p}$, $\mathcal{H}_{a c}$, and $\mathcal{H}_{c s}$, respectively. They are called the pure point, absolutely continuous, and continuous singular components of $\mathcal{G}$. One can also define the absolutely continuous, continuous singular, and pure point components of the spectrum of $\mathcal{G}: \sigma_{a c}(\mathcal{G}):=\sigma\left(\mathcal{G}_{a c}\right), \sigma_{c s}(\mathcal{G}):=\sigma\left(\mathcal{G}_{c s}\right)$ and $\sigma_{p p}(\mathcal{G}):=\sigma\left(\mathcal{G}_{p p}\right)$. The pure point spectrum so defined is the closure of the pure point spectrum defined previously as the actual set of all eigenvalues. Both definitions are used in the literature. With the definition of the pure point spectrum as the closure of the set of all eigenvalues, one has a decomposition: $\sigma(\mathcal{G})=\sigma_{a c}(\mathcal{G}) \cup \sigma_{c s}(\mathcal{G}) \cup \sigma_{p p}(\mathcal{G})$ (however, the three components do not have to be disjoint). The continuous spectrum is $\sigma_{c}(\mathcal{G})=\sigma_{a c}(\mathcal{G}) \cup \sigma_{c s}(\mathcal{G})$.

- We say that $\lambda \in \sigma(\mathcal{G})$ is the essential spectrum of $\mathcal{G}, \sigma_{e}(\mathcal{G})$, if and only if the range of $\mathcal{E}((\lambda-\epsilon, \lambda+\epsilon))$ is infinite-dimensional for all $\epsilon>0$. If $\lambda \in \sigma(\mathcal{G})$, but the range of $\mathcal{E}((\lambda-\epsilon, \lambda+\epsilon))$ is finite-dimensional for some $\epsilon>0$, we say that $\lambda \in \sigma_{d}(\mathcal{G})$, the discrete spectrum of $\mathcal{G}$. This gives a decomposition of the spectrum into two disjoint components, $\sigma(\mathcal{G})=\sigma_{d}(\mathcal{G}) \cup \sigma_{e}(\mathcal{G})$. The set $\sigma_{d}(\mathcal{G})$ is not necessarily closed, but $\sigma_{e}(\mathcal{G})$ is always closed. 
- $\lambda \in \sigma_{d}(\mathcal{G})$ if and only if $\lambda$ is an isolated point of $\sigma(\mathcal{G})$ and $\lambda$ is an eigenvalue of finite multiplicity. Obviously, $\sigma_{d}(\mathcal{G}) \subset \sigma_{p p}(\mathcal{G})$.

- $\lambda \in \sigma_{e}(\mathcal{G})$ if and only if one or more of the following holds: (a) $\lambda \in \sigma_{c}(\mathcal{G}) \equiv$ $\sigma_{a c}(\mathcal{G}) \cap \sigma_{c s}(\mathcal{G})$; (b) $\lambda$ is a limit point of the point spectrum $\sigma_{p}(\mathcal{G})$; (c) $\lambda$ is an eigenvalue of infinite multiplicity.

- Some authors (e.g., [22, p. 1393]) define the essential spectrum as follows: $\sigma_{e}(\mathcal{G}):=\{\lambda \in \mathbb{R}$ : Range of $(\lambda I-\mathcal{G})$ is not closed $\}$. In this definition, the essential spectrum of a self-adjoint operator $\mathcal{G}$ is the set of non-isolated points of $\sigma(\mathcal{G})\left(\left[22\right.\right.$, p. 1395]), and $\lambda \in \sigma_{e}(\mathcal{G})$ if and only if one or more of the following holds: (a) $\lambda \in \sigma_{c}(\mathcal{G}) \equiv \sigma_{a c}(\mathcal{G}) \cap \sigma_{c s}(\mathcal{G})$; (b) $\lambda$ is a limit point of the point spectrum $\sigma_{p}(\mathcal{G})$. Eigenvalues of ordinary differential operators always have finite multiplicity, so for such operators the two definitions of the essential spectrum coincide.

Example. Let $\mathcal{G}$ be a self-adjoint non-positive operator with purely discrete spectrum $\sigma_{d}(\mathcal{G}) \subset(-\infty, 0]$. Then the resolution of the identity can be defined by

$$
\mathcal{E}(B)=\sum_{\lambda \in B} P(\lambda),
$$

where $P(\lambda)$ is the orthogonal projection onto the eigenspace corresponding to the eigenvalue $\lambda \in \sigma_{d}(\mathcal{A})$, i.e., the eigenspace $\operatorname{Ker}(\mathcal{G}-\lambda I)$. Then the spectral theorem takes the simpler form:

$$
\begin{aligned}
P_{t} f=e^{t \mathcal{G}} f & =\sum_{\lambda \in \sigma_{d}(\mathcal{G})} e^{\lambda t} P(\lambda) f, \quad t \geq 0, \quad f \in \mathcal{H}, \\
\mathcal{G} f & =\sum_{\lambda \in \sigma_{d}(\mathcal{G})} \lambda P(\lambda) f, \quad f \in D(\mathcal{G}) .
\end{aligned}
$$

For $t=0$ we have the spectral expansion for any $f \in \mathcal{H}$ :

$$
f=\sum_{\lambda \in \sigma_{d}(\mathcal{G})} P(\lambda) f, \quad\|f\|^{2}=\sum_{\lambda \in \sigma_{d}(\mathcal{G})}\|P(\lambda) f\|^{2} .
$$

If $\left\{\varphi_{n}\right\}$ is a complete orthonormal system of eigenvectors of $\mathcal{G}$, then the Fourier expansion and the Parseval equality are valid for any $f \in \mathcal{H}$ :

$$
f=\sum_{n} c_{n} \varphi_{n}, \quad c_{n}=\left(f, \varphi_{n}\right), \quad\|f\|^{2}=\sum_{n} c_{n}^{2},
$$

and the spectral expansions for the semigroup and the resolvent are:

$$
\begin{aligned}
P_{t} f & =\sum_{n} c_{n} e^{\lambda_{n} t} \varphi_{n}, \quad t \geq 0, \quad f \in \mathcal{H}, \\
R_{\alpha} f & =\sum_{n} \frac{c_{n} \varphi_{n}}{\alpha-\lambda_{n}}, \quad \alpha \in \rho(\mathcal{G}), \quad f \in \mathcal{H} .
\end{aligned}
$$




\section{Acknowledgment}

This paper is based on research supported by the National Science Foundation under grants DMI-0200429 and DMS-0223354.

\section{References}

[1] M. Abramowitz and I. A. Stegun, Handbook of Mathematical Functions (Dover, New York, 1972).

[2] D.-H. Ahn and B. Gao, A parametric nonlinear model of term structure dynamics, Review of Financial Studies 12 (1999) 721-762.

[3] C. Albanese, G. Campolieti, P. Carr and A. Lipton, Black-Scholes goes hypergeometric, Risk (December 2001) 99-103.

[4] D. R. Beaglehole and M. Tenney, A non-linear equilibrium model of the term structure of interest rates: Corrections and additions, Journal of Financial Economics 32 (1992) 345-354.

[5] A. N. Borodin and P. Salminen, Handbook of Brownian Motion (Birkhauser, Boston, 1996).

[6] P. P. Boyle, M. Broadie and P. Glasserman, Monte carlo methods for security pricing, Journal of Economic Dynamics and Control 21 (1997) 1267-1321.

[7] P. P. Boyle and Y. Tian, Pricing lookback and barrier options under the CEV process, Journal of Financial and Quantitative Analysis 34 (1999) 241-264.

[8] M. Brennan and E. Schwartz, A continuous-time approach to the pricing of bonds, Journal of Banking and Finance 3 (1979) 133-155.

[9] Buchholz, The Confluent Hypergeometric Function (Springer, Berlin, 1969).

[10] P. Carr, A. Lipton and D. Madan, The reduction method for valuing derivative securities, working paper (2002), http://www.math.nyu.edu/research/carrp/papers/ pdf/symmetry17.pdf.

[11] E. Coddington and N. Levinson, Theory of Ordinary Differential Equations (McGraw-Hill, New York, 1955).

[12] A. Comtet, C. Monthus and M. Yor, Exponential functionals of Brownian motion and disordered systems, Journal of Applied Probability 35 (1998) 255-271.

[13] J. C. Cox, Notes on option pricing I: Constant elasticity of variance diffusions, working paper (1975) Stanford University (reprinted in Journal of Portfolio Management 22 (1996) $15-17$.

[14] J. C. Cox, J. E. Ingersoll and S. A. Ross, A theory of the term structure of interest rates, Econometrica 53 (1985) 385-407.

[15] J. C. Cox and S. Ross, The valuation of options for alternative stochastic processes, Journal of Financial Economics 3 (1976) 145-166.

[16] E. B. Davies, One-Parameter Semigroups (Academic Press, London, 1980).

[17] D. Davydov and V. Linetsky, The valuation and hedging of barrier and lookback options under the CEV process, Management Science 47 (2001) 949-965.

[18] D. Davydov and V. Linetsky, Structuring, pricing and hedging double barrier step options, Journal of Computational Finance 5 Winter (2002) 55-87.

[19] D. Davydov and V. Linetsky, Pricing options on scalar diffusions: An eigenfunction expansion approach, Operations Research 51 (2003) 185-209.

[20] C. Delacherie and P.-A. Meyer, Probabilities and Potential C. Potential Theory for Discrete and Continuous Semigroups (North-Holland, Amsterdam, 1988).

[21] C. Donati-Martin, R. Ghomrasni and M. Yor, On certain markov processes attached to exponential functionals of brownian motion: Applications to asian options, Revista Matematica Iberoamericana Madrid 17(1) (2001) 179-193. 
[22] N. Dunford and J. Schwartz, Linear Operators. Part II: Spectral Theory (Self-Adjoint Operators in Hilbert Spaces) (Wiley, NJ, 1963).

[23] J. Elliott, Eigenfunction expansions associated with singular second order differential operators, Transactions of the American Mathematical Society 78 (1955) 406-425.

[24] A. Erdelyi, Higher Transcendental Functions Vol. II (MacGraw-Hill, New York, 1953).

[25] W. Feller, Two singular diffusion problems, Annals of Mathematics 54 (1951) 173182.

[26] J.-P. Florens, E. Renault and N. Touzi, Testing for embeddability by stationary reversible continuous-time markov processes, Econometric Theory 14 (1998) 744769.

[27] M. Fukushima, Y. Oshima and M. Takeda, Dirichlet Forms and Symmetric Markov Processes (W. de Gruyter, Berlin, 1994).

[28] C. Fulton, S. Pruess and Y. Xie, The automatic classification of Sturm-Liouville problems, preprint, http://www.mines.edu/fs_home/spruess/papers/class.ps.

[29] H. Geman and M. Yor, Bessel processes, asian options and perpetuities, Mathematical Finance 3 (1993) 349-75.

[30] H. Geman and M. Yor, Pricing and hedging double barrier options: A probabilistic approach, Mathematical Finance 6 (1996) 365-78.

[31] I. Glazman, Direct Methods of Qualitative Spectral Analysis of Singular Differential Operators, English Translation (Monson, Jerusalem, 1965).

[32] A. Goeing-Jaeschke and M. Yor, A survey and some generalizations of bessel processes, Bernoulli 9 (2003) 313-349.

[33] R. Goldstein and W. P. Keirstead, On the term structure of interest rates in the presence of reflecting and absorbing boundaries, working paper, Ohio State University (1997), http://fisher.osu.edu/fin/journal/dice/papers/1997/97-1.html.

[34] V. Gorovoi and V. Linetsky, Black's model of interest rates as options, eigenfunction expansions and Japanese interest rates, Mathematical Finance 14 (2004) 49-78.

[35] I. S. Gradshteyn and I. M. Ryzhik, Tables of Integrals, Series and Products (Academic Press, New York, 1994).

[36] C. Grosche and F. Steiner, Handbook of Feynman Path Integrals (Springer, Berlin, 1998).

[37] L. P. Hansen, J. Scheinkman and N. Touzi, Spectral methods for identifying scalar diffusions, Journal of Econometrics 86 (1998) 1-32.

[38] H. He, W. Keirstead and J. Rebholz, Double lookbacks, Mathematical Finance 8 (1998) 201-228.

[39] S. L. Heston, A closed-form solution for options with stochastic volatility with applications to bond and currency options, Review of Financial Studies 6 (1993) 327-343.

[40] S. L. Heston, A simple new formula for options with stochastic volatility, working paper (Washington University, 1997).

[41] E. Hille and R. S. Phillips, Functional Analysis and Semigroups (American Mathematical Society, Providence, RI, 1957).

[42] K. Ito and H. McKean, Diffusion Processes and their Sample Paths, 2nd Printing (Springer, Berlin, 1974).

[43] J. C. Jackwerth and M. Rubinstein, Recovering stochastic processes from option prices, working paper, University of California, Berkeley (1998).

[44] F. Jamshidian, Bond, futures and option evaluation in the quadratic interest rate model, Applied Mathematical Finance 3 (1996) 93-115.

[45] S. Karlin and J. McGregor, The differential equations of birth-and-death processes and the stieltjes moment problem, Transactions of American Mathematical Society 85 (1957) 489-546. 
[46] S. Karlin and H. M. Taylor, A Second Course in Stochastic Processes (Academic Press, San Diego, 1981).

[47] D. G, Kendall, Pole-seeking brownian motion and bird navigation, Journal of Royal Statistical Society B36 (1972) 365-417.

[48] J. Kent, Eigenvalue expansions for diffusion hitting times, Z. Wahrsch. Verw. Gebiete 52 (1980) 309-319.

[49] J. Kent, The spectral decomposition of a diffusion hitting time, Annals of Applied Probability 10 (1982) 207-19.

[50] N. Kunitomo and M. Ikeda, Pricing options with curved boundaries, Mathematical Finance 2 (1992) 275-98.

[51] H. Langer and W. S. Schenk, Generalized second-order differential operators, corresponding gap diffusions and superharmonic transformations, Math. Nachr. 148 (1990) 7-45.

[52] K. S. Larsen and M. Sorensen, Diffusion models for exchange rates in a target zone, working paper, University of Copenhagen (2003).

[53] N. Levinson, A simplified proof of the expansion theorem for singular second order differential operators, Duke Mathematical Journal 18 (1951) 57-71.

[54] B. M. Levitan, Expansion in characteristic functions of differential equations of the second order (in Russian), Gostekhizdat, Moscow (1950).

[55] B. M. Levitan and I. S. Sargsjan, Introduction to Spectral Theory (American Mathematical Society, Providence, RI, 1975).

[56] A. Lewis, Three expansion regimes for interest rate term structure models, working paper, (1994) http://www.optioncity.net/publications.html.

[57] A. Lewis, Applications of eigenfunction expansions in continuous-time finance, Mathematical Finance 8 (1998) 349-383.

[58] A. Lewis, Option Valuation under Stochastic Volatility (Finance Press, CA, 2000).

[59] V. Linetsky, Step options, Mathematical Finance 9 (1999) 55-96.

[60] V. Linetsky, Spectral expansions for Asian (average price) options, to appear in Operations Research.

[61] V. Linetsky, Exotic spectra, Risk (April 2002) pp. 85-89.

[62] V. Linetsky, Lookback options and hitting times of one-dimensional diffusions: A spectral expansion approach, Finance and Stochastics 8(3) (2004).

[63] V. Linetsky, The spectral representation of Bessel processes with drift: Applications in queueing and finance, Journal of Applied Probability 41(2) (2004) 327-344.

[64] V. Linetsky, Computing hitting time densities for CIR and OU diffusions: Applications to mean-reverting models, Journal of Computational Finance 7(4) (2004).

[65] A. Lipton, Similarities via self-similarities, Risk (September 1999) pp. 101-105.

[66] A. Lipton, Mathematical Methods for Foreign Exchange (World Scientific, Singapore, 2001).

[67] A. Lipton, The volatility smile problem, Risk (February 2002) pp. 61-65.

[68] A. Lipton and W. McGhee, Universal barriers, Risk (May 2002) pp. 81-85.

[69] H. McKean, Elementary solutions for certain parabolic partial differential equations, Transactions of the American Mathematical Society 82 (1956) 519-548.

[70] R. C. Merton, Theory of rational option pricing, Bell J. Econ. Mgmt. Sci. 4 (1973) 141-183.

[71] R. Merton, An asymptotic theory of growth under uncertainty, Review of Economic Studies 42 (1975) 375-393.

[72] P. M. Morse, Diatomic molecules according to the wave mechanics. II. Vibrational levels, Physical Review 34 (1929) 57. 
[73] P. M. Morse and H. Feshbach, Methods of Theoretical Physics, Part II (McGraw-Hill, 1953).

[74] D. B. Nelson, ARCH models as diffusion approximations, Journal of Econometrics 45 (1990) 7-39.

[75] A. Pelsser, Pricing double barrier options using analytical inversion of laplace transforms, Finance and Stochastics 4 (2000) 95-104.

[76] D. Pilipović, Energy Risk (McGraw-Hill, New York, 1998).

[77] J. W. Pitman and M. Yor, Bessel processes and infinitely divisible laws, in Stochastic Integrals, Lecture Notes in Mathematics, Vol. 851 (Springer, 1981).

[78] J. W. Pitman and M. Yor, A decomposition of Bessel bridges, Z. Wahrscheinlichkeit. 59 (1982) 425-457.

[79] J. D. Pryce, Numerical Solution of Sturm-Liouville Problems (Clarendon, Oxford, 1993).

[80] A. P. Prudnikov, Yu. A. Brychkov and O. I. Marichev, Integrals and Series Vol. 2 (Gordon and Breach, New York, 1986).

[81] M. Reed and B. Simon, Functional Analysis (Academic Press, San Diego, 1980).

[82] T. Shiga and S. Watanabe, Bessel diffusions as a one-parameter family of diffusion processes, Z. Wahrscheinlichkeit. Verw. 27 (1973) 37-46.

[83] L. J. Slater, Confluent Hypergeometric Functions (Cambridge University Press, 1960).

[84] E. C. Titchmarsh, Eigenfunction Expansions Associated with Second-order Differential Equations (Clarendon, Oxford, 1962).

[85] O. A. Vasicek, An equilibrium characterization of the term structure, Journal of Financial Economics 5 (1977) 177-188.

[86] S. Watanabe, On time inversion of one-dimensional diffusion processes, $Z$. Wahrscheinlichkeit. 31 (1975) 115-124.

[87] J. Weidmann, Spectral Theory of Ordinary Differential Operators, Lecture Notes in Mathematics, Vol. 1258 (Springer, Berlin, 1987).

[88] E. Wong, The construction of a class of stationary Markoff processes, in Sixteenth Symposium in Applied Mathematics - Stochastic Processes in Mathematical Physics and Engineering, ed. R. Bellman (American Mathematical Society, Providence, RI, 1964) pp. 264-276.

[89] M. Yor, On square-root boundaries for Bessel processes, and pole-seeking Brownian motion, in Stochastic Analysis and Applications, Lecture Notes in Mathematics, Vol. 1095 (1984) pp. 100-107.

[90] M. Yor, Exponential Functionals of Brownian Motion and Related Processes (Springer, Berlin, 2001). 
Copyright of International Journal of Theoretical \& Applied Finance is the property of World Scientific Publishing Company and its content may not be copied or emailed to multiple sites or posted to a listserv without the copyright holder's express written permission. However, users may print, download, or email articles for individual use. 